ARTICLE

\title{
Defining the therapeutic selective dependencies for distinct subtypes of PI3K pathway-altered prostate cancers
}

Ninghui Mao ${ }^{1,10}$, Zeda Zhang 1,2,10, Young Sun Lee ${ }^{1}$, Danielle Choi ${ }^{1}$, Aura Agudelo Rivera ${ }^{1}$, Dan Li $\mathbb{D}^{1}$, Cindy Lee Samuel Haywood ${ }^{3,4}$, Xiaoping Chen ${ }^{5}$, Qing Chang ${ }^{5}$, Guotai Xu1, Hsuan-An Chen (1) ${ }^{2}$, Elisa de Stanchina ${ }^{5}$,

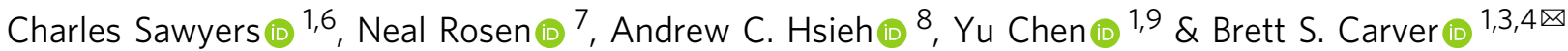

Previous studies have suggested that PTEN loss is associated with p110 $\beta$ signaling dependency, leading to the clinical development of p110 $\beta$-selective inhibitors. Here we use a panel pre-clinical models to reveal that PI3K isoform dependency is not governed by loss of PTEN and is impacted by feedback inhibition and concurrent PIK3CA/PIK3CB alterations. Furthermore, while pan-PI3K inhibition in PTEN-deficient tumors is efficacious, upregulation of Insulin Like Growth Factor 1 Receptor (IGF1R) promotes resistance. Importantly, we show that this resistance can be overcome through targeting AKT and we find that AKT inhibitors are superior to pan-PI3K inhibition in the context of PTEN loss. However, in the presence of wildtype PTEN and PIK3CA-activating mutations, p110 $\alpha$-dependent signaling is dominant and selectively inhibiting $p 110 \alpha$ is therapeutically superior to AKT inhibition. These discoveries reveal a more nuanced understanding of PI3K isoform dependency and unveil novel strategies to selectively target PI3K signaling nodes in a context-specific manner.

\footnotetext{
${ }^{1}$ Human Oncology and Pathogenesis Program, Memorial Sloan Kettering Cancer Center, New York, NY, USA. ${ }^{2}$ Louis V. Gerstner Jr. Graduate School of Biomedical Sciences, Memorial Sloan Kettering Cancer Center, New York, NY, USA. ${ }^{3}$ Department of Surgery, Memorial Sloan Kettering Cancer Center, New York, NY, USA. ${ }^{4}$ Department of Urology, Memorial Sloan Kettering Cancer Center, New York, NY, USA. ${ }^{5}$ Antitumor Assessment Core Facility, Memorial Sloan Kettering Cancer Center, New York City, NY, USA. ${ }^{6}$ Howard Hughes Medical Institute, Memorial Sloan Kettering Cancer Center, New York, NY, USA. ${ }^{7}$ Molecular Pharmacology Program, Memorial Sloan Kettering Cancer Center, New York, NY, USA. ${ }^{8}$ Divisions of Human Biology and Clinical Research, Fred Hutchinson Cancer Research Center, Seattle, WA, USA. ${ }^{9}$ Department of Medicine, Memorial Sloan Kettering Cancer Center, New York, NY, USA. ${ }^{10}$ These

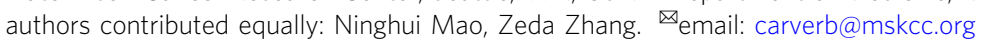


T he phosphoinositide 3-kinase (PI3K) pathway is one of the most altered oncogenic pathways in human cancer $^{1,2}$. In hormone-regulated cancers such as prostate and breast cancer, activation of the PI3K pathway is often driven by mutations/amplification/overexpression of PIK3CA/PIK3CB, or loss of the tumor suppressor $P T E N^{3-6}$. According to recent cancer genomic sequencing efforts, PIK3CA alterations are found in more than $30 \%$ of breast cancers, and PIK3CA/PIK3CB alterations are observed in $15 \%$ of prostate cancers ${ }^{3-6}$. Loss of PTEN occurs in approximately $40 \%$ of metastatic prostate cancers and represents the most common alteration in the PI3K pathway ${ }^{3,4}$. Previous studies using preclinical cell lines and xenograft models have shown that targeting PI3K activity could be a promising therapeutic strategy to treat tumors with PI3K pathway alterations $s^{7,8}$. However, early attempts to develop PI3K inhibitors did not advance clinically due to nonselective target inhibition, toxicity necessitating dose reductions, and the recently described concept of relief of feedback inhibition, all of which reduced efficacy ${ }^{9,10}$.

The PI3K holoenzyme is composed of a catalytic subunit (p110) and a regulatory subunit (p85, p55, and p50). In epithelial lineages, $\mathrm{p} 110 \alpha(P I K 3 C A)$ and $\mathrm{p} 110 \beta$ (PIK3CB) are the dominantly expressed catalytic subunit isoforms ${ }^{8,11}$. Oncogenic activity of PI3K-altered tumors is often dependent on different p110 isoforms ${ }^{12-16}$. In breast cancer, activating PIK3CA mutations are dominant drivers of PI3K signaling whereas in PTENdeficient prostate cancer, such activity is reported to be driven by $\mathrm{p} 110 \beta^{16,17}$. These findings subsequently propelled the development of the next generation of agents designed to target individual p110 isoforms in a selective manner ${ }^{18-20}$.

Previous studies have documented a reciprocal negative feedback regulation between PI3K activity and hormone receptor (e.g. androgen receptor) signaling in hormone-dependent cancers including breast and prostate cancer ${ }^{21-24}$. This work provided the molecular rationale for the development of clinical trials cotargeting hormone receptor and PI3K signaling in PI3K pathwayaltered breast or prostate tumors. A phase II trial of combining a second-generation AR antagonist and an inhibitor of AKT, a downstream component of the PI3K pathway, demonstrated significant improvement in radiographic progression-free survival (RPFS) for metastatic prostate cancer patients with tumors displaying loss of $P T E N^{25}$. The results of this trial have led to the accrual of a phase III study that recently met its co-primary endpoint in PTEN-loss metastatic castration resistant prostate cancer (mCRPC) demonstrating a significant improvement in RPFS. Additionally, the recent phase III SOLAR1 trial reported a significant improvement in progression-free survival for breast cancer patients harboring PIK3CA mutations when treated with BYL719 (p110a inhibitor) plus fulvestrant (ER degrader) ${ }^{14,26-28}$. In prostate cancer, while previous studies suggest that loss of PTEN is associated with p110 $\beta$ dependency and early phase clinical trials have been developed to evaluate the efficacy of p110 3 -selective inhibitors in this context, several caveats to this approach remain. First, the original studies reporting that loss of $P T E N$ promoted $\mathrm{p} 110 \beta$ dominant signaling were performed in a limited number of models. Secondly, investigators have shown that in the context of PTEN loss, inhibition of p110 $\beta$ leads to relief of feedback inhibition of the non-dominant p110 isoforms resulting in PI3K rebound signaling and diminished efficacy ${ }^{29}$. Despite these caveats, enthusiasm for p110 isoform-selective inhibitors for the management of PI3K pathway-altered prostate cancer continues, with the hope of maximizing efficacy and minimizing toxicity.

Here, we analyze a broad collection of prostate cancer models to identify the appropriate molecular context and therapeutic strategies to selectively target PI3K pathway-altered prostate cancers. We define the impact of PTEN status and PI3K alterations on p110 isoform dependency, further study the role of relief of feedback between p110 isoforms across the common PI3K pathway alterations and reveal a novel and actionable mechanism of resistance to PI3K inhibition.

\section{Results}

Loss of PTEN does not drive p110 $\beta$ dependency. Alterations in the PI3K pathway (PTEN, PIK3CA, PIK3CB, and PIK3R1) are seen in more than $50 \%$ metastatic castration-resistant prostate cancer (mCRPC) (Fig. 1a). Among these cases, the majority (82\%) harbor PTEN deletion or mutation, while others ( 11-19\%) display amplification, mutations, or mRNA upregulation in PIK3CA or PIK3CB (Fig. 1b). Additionally, further characterization reveals a significant co-occurrence between $P T E N$ and PIK3CA/PIK3CB/PIK3R1 alterations despite their epistatic relationship (Fig. 1c). This high rate of co-occurrence indicates that concomitant alterations in members of the PI3K pathway may have additive or synergistic roles in prostate tumorigenesis.

To systematically model PI3K pathway alterations and study p110 isoform dependencies in mCRPC, we evaluated a broad collection of patient-derived prostate cancer organoids (PDOs) established from metastatic tumor biopsies of mCRPC patient ${ }^{30}$. We also analyzed two previously established prostate cancer cell lines, LNCaP (PTEN loss) and CWR22Pc (PIK3CA Q546R). These prostate cancer models harbor various alterations in the PI3K pathway and exhibit differential PI3K signaling activity, as shown by the levels of downstream AKT phosphorylation (Fig. 1d, e). Focusing on the common regulators of PI3K signaling, we observed a high frequency of genomic deletion of PTEN across the pre-clinical models, one cell line with a PIK3CA activating mutation, and three with alterations of PIK3R1. While $P I K 3 C B$ and $A K T$ activating mutations occur at a low frequency in $\mathrm{mCRPC}$, we did not identify alterations or activating mutations of these genes in our models.

PI3K signaling in PTEN-deficient prostate cancer has been reported to be dependent on p110 $\beta$, but the models evaluated were limited in number ${ }^{12,13,15}$. Using our collection of PDOs, we first tested if their PI3K activity is dependent on p110 $\alpha$ or p110 $\beta$ by treating them with a p110 $\alpha$-selective inhibitor (BYL719) or a p110ß-selective inhibitor (AZD8186). We used both compounds at their on-target dosage that does not cross-inhibit other PI3K isoforms ${ }^{1}$. Surprisingly, despite their PTEN deficiency, MSKPCa3, MSK-PCa11, and MSK-PCa8 showed selective sensitivity to $\mathrm{p} 110 \alpha$ inhibition (BYL719) rather than $\mathrm{p} 110 \beta$ inhibition (AZD8186), while MSK-PCa1 displayed p110 $\beta$ dependency similar to LNCaP (Fig. 1f). Since organoid culture media contains various growth factors, which may bias PI3K activation through receptor tyrosine kinase (RTK)-mediated $\mathrm{p} 110 \alpha$ activation, we next tested if p110 or p110 $\beta$ dependency is influenced by the components in media. We switched the regular 10\% FBS RPMI media with organoid culture media for $48 \mathrm{~h}$ and observed that PI3K isoform dependency remained the same in both p110 $\beta$ dependent LNCaP and p110a-dependent CWR22Pc (Supplementary Fig. 1A, B). Furthermore, we evaluated the concept that PTEN loss causes p110 $\beta$ dependency in prostate cancer. To accomplish this, we performed CRISPR-Cas9 knockout of PTEN in two PTEN wild-type prostate cancer organoid models and the PTEN wild-type CWR22Pc cell line. When PTEN was deleted we observed that all three models remain predominantly dependent on $\mathrm{p} 110 \alpha$, not on p110 (Fig. $1 \mathrm{~g}$ and Supplementary Fig. 1C). Conversely, to evaluate the effect of PTEN expression on p110 $\beta$ dependency, we ectopically expressed wild-type PTEN in LNCaP cells using a doxycycline-inducible Tet-on system. Indeed, we 
a

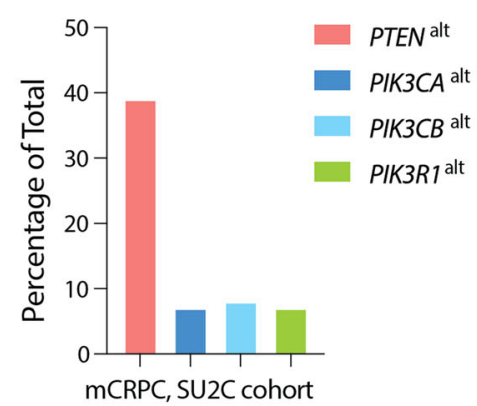

C

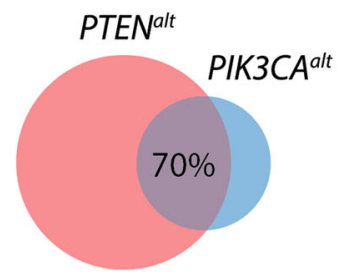

d

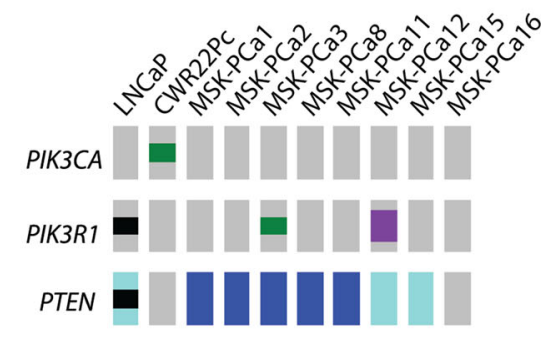

No Alterations Deep Deletion Shallow Deletion

【 Fusion Missense Mutation (Putative driver)

- Truncating Mutation (putative driver)

f b

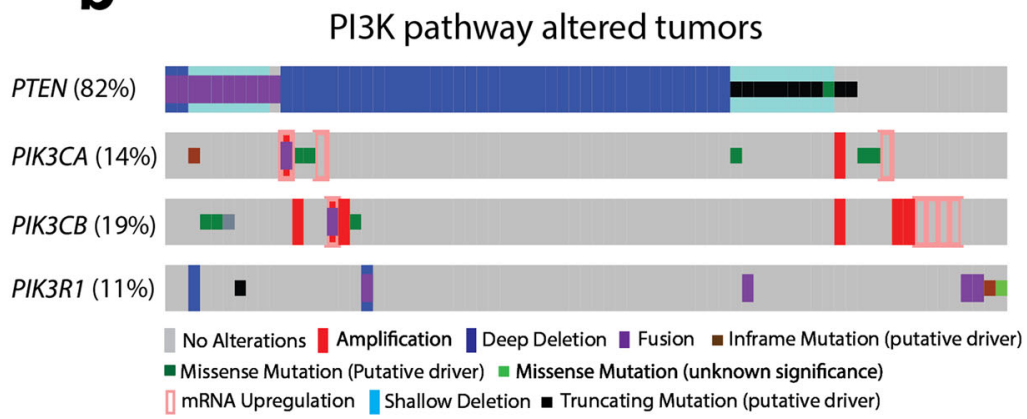

PTEN alt
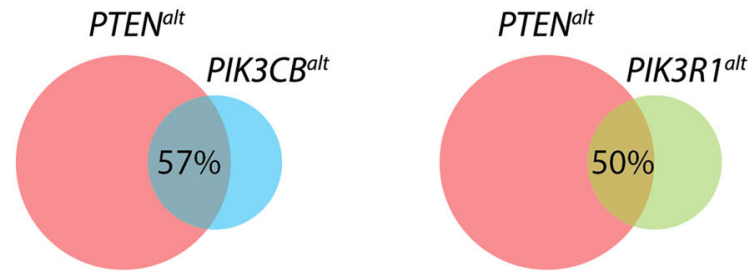

e
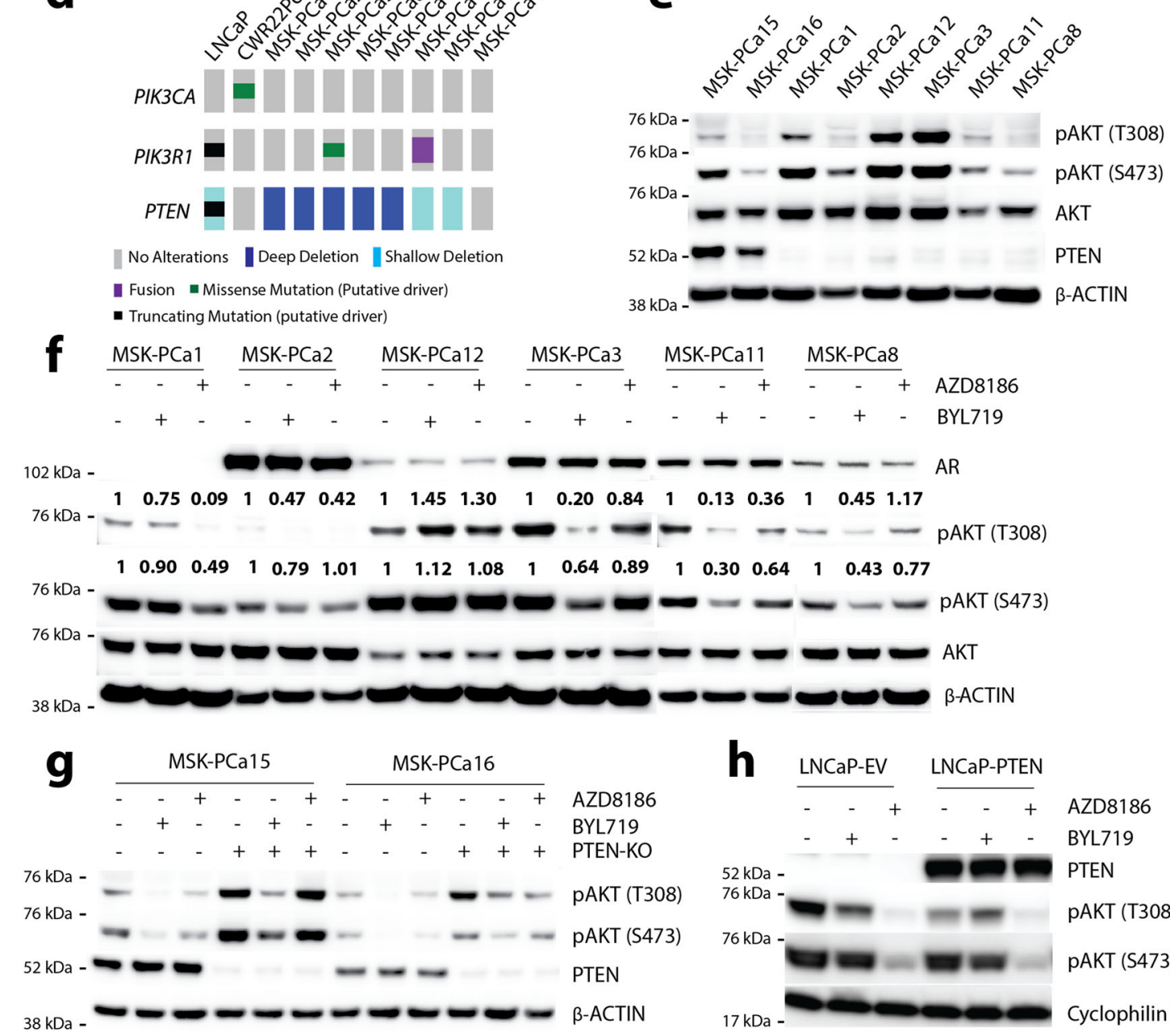

detected a reduction of PI3K activity, however, those LNCaPPTEN cells retained their inherent p110 $\beta$ dependency (Fig. 1h). Collectively, using ex vivo PDOs and in vitro cell line models, we showed that $\mathrm{p} 110 \alpha$ or $\mathrm{p} 110 \beta$ dependency is not solely determined by PTEN status. These results highlight that loss of PTEN is not a dominant biomarker for $\mathrm{p} 110 \beta$-selective signaling and raises the need to further define the mechanisms that drive p110 isoform

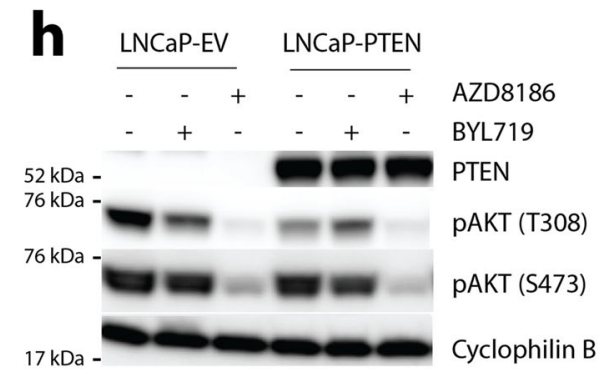

dependency. This discovery carries clinical significance as the development of p110 $\beta$ isoform-selective inhibitors has been focused on cancers harboring loss of PTEN.

Activating PIK3CA/PIK3CB mutations determine p110 isoform dependency in prostate cancer. We next sought to investigate if there is a clinically relevant mechanism that could shape 
Fig. 1 Modeling PI3K alterations using patient-derived organoid (PDO) reveals PI3K isoform dependency is not determined by PTEN. a Percentage of PI3K members (PTEN, PIK3CA, PIK3CB, and PIK3R1) alterations in metastatic castration-resistant prostate cancer cohort (SU2C Cell 2015). b Oncoprint showing PI3K member alterations in all PI3K-altered tumors from the same cohort $(n=76)$. $\mathbf{c}$ Venn diagram showing the percentage of overlaps among alterations in PIK3CA, PIK3CB, PIK3R1, and PTEN. d Oncoprint showing PIK3CA, PIK3R1, and PTEN alterations in a collection of PDOs. e Western blot showing pAKT level and PTEN status in a collection of PDOs. $\mathbf{f}$ Western blot showing AR and pAKT levels in PDOs treated with BYL719 (1 $\mu$ M), AZD8186 (250 nM), or Veh for $4 \mathrm{~h}$. Quantification of pAKT473 and pAKT308 normalized to $\beta$-actin. $\mathbf{g}$ Western blot showing pAKT levels in isogenic PDO pairs MSK-PCa15 (PTEN-intact, PTEN-KO) and MSK-PCa16 (PTEN-intact, PTEN-KO) treated with BYL719 (1 $\mu$ M), AZD8186 (250 nM), or Veh for $4 \mathrm{~h}$. h Western blot showing pAKT levels in isogenic LNCaP-EV and LNCaP-PTEN pairs treated with doxycycline $(500 \mathrm{ng} / \mathrm{mL})$ for $8 \mathrm{~h}$ for PTEN induction, followed by either BYL719 $(1 \mu \mathrm{M})$, AZD8186 $(250 \mathrm{nM})$, or Veh for $4 \mathrm{~h}$. All assays were performed with three biological replicates. Source data are provided as Source data file.

p110 isoform dependency in prostate tumors. By analyzing whole-exome sequencing results from an advanced prostate cancer patient cohort, we explored potentially activating alterations in the PI3K pathway and identified oncogenic alterations across the dominant drivers of PI3K signaling (PTEN, PIK3CA, PIK3CB, PIK3R1) (Fig. 1a). Interestingly, while activating alterations in PIK3CA/PIK3CB can occur in the setting of wildtype $P T E N$, the alterations were more frequently enriched in $P T E N$-altered tumors (Fig. 1b, c). Based on this observation, we hypothesized that gain-of-function alterations in PIK3CA or PIK3CB could work synergistically with PTEN deficiency to further enhance oncogenic PI3K signaling and shape p110 isoform dependency. To this end, we expressed $\mathrm{p} 110 \alpha^{\mathrm{E} 545 \mathrm{~K}}$ or p1 $10 \beta^{\mathrm{E} 1051 \mathrm{~K}}$ activating mutants in the pre-clinical models (LNCaP, CWR22Pc, and MSK-PCa1) using lentiviral transduction. Ectopic expression of mutant $\mathrm{p} 110 \alpha^{\mathrm{E} 545 \mathrm{~K}}$ or $\mathrm{p} 110 \beta^{\mathrm{E} 1051 \mathrm{~K}}$ further enhanced baseline PI3K activity in both the PTEN-null (LNCaP, MSK-PCa1) and PTEN wild-type (CWR22Pc) backgrounds (Fig. $2 \mathrm{a}-\mathrm{c}$ ). These results suggest that hyper-activation of p110 subunits can further elevate PI3K pathway activity regardless of PTEN status and there is cooperativity among activating alterations in members of the PI3K pathway. We then sought to ask if $\mathrm{p} 110 \alpha / \mathrm{p} 110 \beta$-activating mutations influence $\mathrm{p} 110$ isoform dependency in the PTEN-deficient model LNCaP. Surprisingly, we observed that expression of $\mathrm{p} 110 \alpha^{\mathrm{E} 545 \mathrm{~K}}$ in LNCaP cells shifted their p110 $\beta$ dependency towards more p110 $\alpha$-dependent signaling (Fig. 2d). LNCaP-p110a ${ }^{\text {E545K }}$ cells were also no longer sensitive to AZD8186 (p110 $\beta$ inhibition) at its on-target or higher concentration dosing in in vitro growth assays (Fig. 2e, Supplementary Fig. 2A). Importantly, while standard dosing of BYL719 ( $\mathrm{p} 110 \mathrm{a}$ inhibition) was not sufficient to inhibit cell proliferation, to account for the exogenous $\mathrm{p} 110 \alpha^{\mathrm{E} 545 \mathrm{~K}}$ overexpression, we indeed found that increasing the concentration of BYL719 suppressed cell growth (Fig. 2e, Supplementary Fig. 3A). Similarly, in the PTEN wild-type model CWR22Pc, expression of $\mathrm{p} 110 \beta^{\mathrm{E} 1051 \mathrm{~K}}$ shifted its $\mathrm{p} 110 \alpha$ dependency towards more $\mathrm{p} 110 \beta$ dependent signaling (Fig. 2f, g and Supplementary Fig. 2B, C). Additionally, we found that in the context of PTEN loss the overexpression of the activating PIK3CA/PIK3CB mutants did not affect cell proliferation, presumably because PI3K signaling is already robustly activated. However, in the setting of wild-type $P T E N$, expression of these mutants significantly promoted cell proliferation (Fig. 2e, g).

Given that some of the PIK3CA/PIK3CB alterations involve amplification or mRNA upregulation of wild-type alleles, we evaluated if ectopic expression of wild-type PIK3CA impacted PI3K signaling. Indeed, overexpression of $\mathrm{p} 110 \alpha^{\mathrm{WT}}$ in a PTEN deficient (p110 $\beta$-dependent) pre-clinical model promoted PI3K signaling through $\mathrm{p} 110 \alpha$, impacting isoform selective signaling dependency. However, this effect was not as robust as the expression of constitutive activating PIK3CA mutations (Fig. 2h). This suggests that while overexpression of wild-type p110a can promote isoform selective signaling and dependency, activating PIK3CA mutations indeed play a more dominant role. Taken together, we have demonstrated that aberrant PI3K signaling driven by amplification or activating mutations of $P I K 3 C A$ or $P I K 3 C B$ are able to shift p110 isoform dependency regardless of PTEN status across multiple prostate cancer models.

PTEN-deficiency enhances relief of feedback inhibition through $\mathrm{p} 110 \alpha$ and $\mathrm{p} 110 \beta$ signaling following contralateral isoform selective inhibition. Previous studies in prostate cancer harboring loss of PTEN have demonstrated the impact of relief of feedback inhibition and activation of p110a following p110 $\beta$ selective inhibition ${ }^{31,32}$. Furthermore, recent results suggested that in PIK3CA or HER2-mutant breast cancers, loss of PTEN was a driver of resistance to p110a-targeting strategies through the maintenance of PI3K signaling by alternative p110 isoforms ${ }^{33}$. To determine if relief of feedback inhibition was reciprocal between p110 $\alpha$ and $\mathrm{p} 110 \beta$, we performed time-course experiments, using two p110 $\beta$-dependent models, MSK-PCal and LNCaP, and a p110a-dependent model, MSK-PCa3. Similar to our previous studies in LNCaP, following the initial potent response to PI3K signaling inhibition with AZD8186 (p110ß inhibitor), reactivation of PI3K signaling occurred through p110a in MSKPCa1 and LNCaP (Fig. 3a and Supplementary Fig. 3D) ${ }^{31}$. Importantly, for the first time, we were able to show that in a PTEN-deficient prostate cancer model that displays p110a dominance (MSKPCa3), selective inhibition of p110a (BYL719) resulted in feedback activation of PI3K signaling through p110 $\beta$ (Fig. 3c). Feedback activation of PI3K was effectively blocked with dual inhibition of both p110 isoforms. Combined inhibition of p110 $\alpha+$ p110 $\beta$ also achieved the most potent anti-proliferative effect in three independent PDO and cell line models in vitro (Fig. 3b, d and Supplementary Fig. 3C). Our data demonstrate that in the context of PTEN loss, regardless of p110 isoform signaling dominance, targeting both $\mathrm{p} 110 \alpha$ and $\mathrm{p} 110 \beta$ is required to overcome relief of feedback inhibition.

To determine if PTEN status would affect the modulation of feedback, we next used the isogenic pairs of CWR22Pc-sgNT and $-\operatorname{sg} P T E N$ to test the combinatorial effect of p110 a and p110 $\beta$ dual inhibition. In these PIK3CA-mutant, p110a-dependent isogenic pairs, we observed a significantly more robust rebound of PI3K signaling in CWR22Pc-sgPTEN cells as compared to the PTENintact isogenic pair (CWR22Pc-sgNT) following p110a inhibition (Fig. 3e, f). In both CWR22Pc-sgNT and -sgPTEN cells, potent PI3K signaling inhibition was achieved by co-targeting $\mathrm{p} 110 \mathrm{a}$ and p110 (Fig. 3e, f). However, despite combined selective p110 $\alpha$ and p110 $\beta$ inhibition, low levels of rebound signaling through pAKT remains in CWR22Pc-sgPTEN, indicating an incomplete PI3K signaling inhibition even with the combination (Fig. 3f). Furthermore, in our PTEN deficient prostate cancer models (LNCaP, MSKPCa1, and MSKPCa3) a similar phenotype of residual rebound $\mathrm{PI} 3 \mathrm{~K}$ signaling is observed following combined p110 $\alpha$ and p110 $\beta$ inhibition (Fig. 3a, c and Supplementary Fig. 3D). Collectively, this data suggests that targeting AKT directly may be the optimal approach for suppressing downstream PI3K signaling in the molecular context of loss of PTEN. 
a

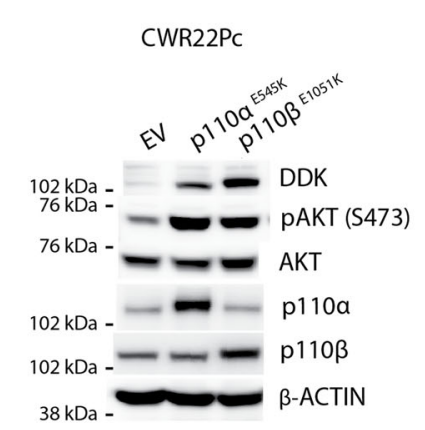

b

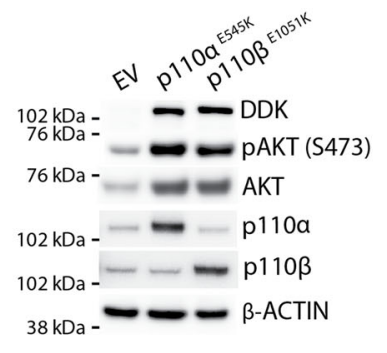

C MSK-PCa1

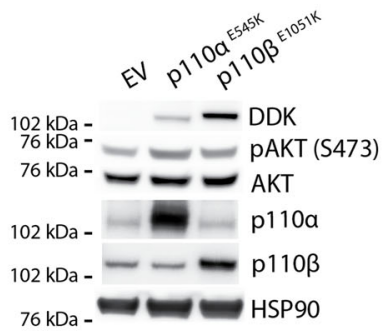

d
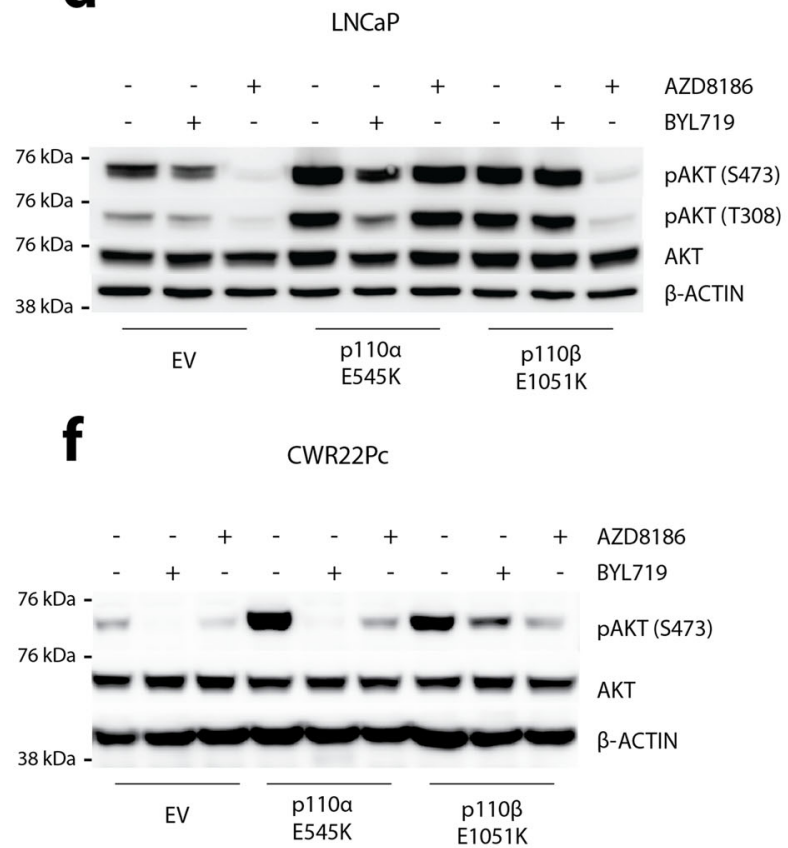

h

e

LNCaP

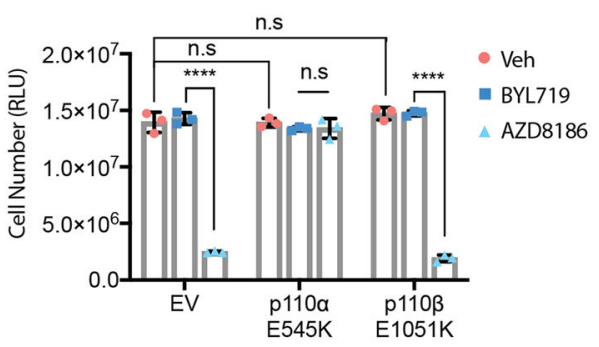

g

CWR22Pc

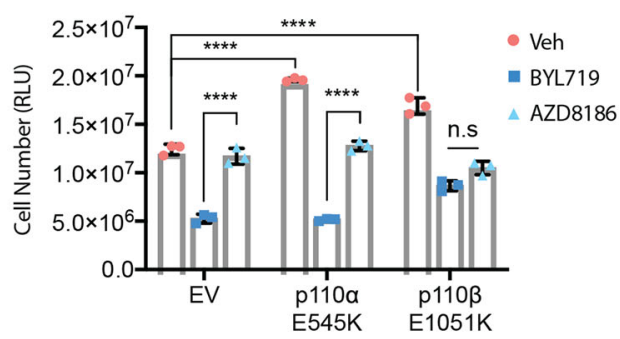

Fig. 2 Activating PIK3CA/PIK3CB mutations determine p110 isoform dependency in prostate cancer. a-c Western blot showing pAKT levels in CWR22Pc, LNCaP cells, or MSK-PCa1 organoids with ectopic expression of DDK-tagged p110 $\alpha^{\text {E545K }}$ or p110 $\beta^{\text {E1051K }}$ mutants. $\mathbf{d}$ Western blot showing pAKT levels in LNCaP-EV, LNCaP-p110 $\alpha^{E 545 K}$, or LNCaP-p110 E1051K cells treated with BYL719 (1 $\mu$ M), AZD8186 (250 nM), or Veh for 4 h. e CellTiter-Glo assay showing cell viability in LNCaP-EV, LNCaP-p110 $\alpha^{\mathrm{E} 545 \mathrm{~K}}$, or LNCaP-p110 $\beta^{\mathrm{E} 1051 \mathrm{~K}}$ cells treated with BYL719 $(1 \mu \mathrm{MM}), \mathrm{AZD} 8186$ (250 nM), or Veh for 7 days. f Western blot showing pAKT levels in CWR22Pc-EV, CWR22Pc-p110 $\alpha^{\text {E545K }}$, or CWR22Pc-p110 $\beta^{E 1051 K}$ cells treated with BYL719 (1 $\mu$ M), AZD8186 $(250 \mathrm{nM})$, or Veh for $4 \mathrm{~h} . \mathbf{g}$ CellTiter-Glo assay showing cell viability in CWR22Pc-EV, CWR22Pc-p110 $\alpha$ E545K, or CWR22Pc-p110 $\beta^{\text {E1051K }}$ cells treated with BYL719 $(1 \mu \mathrm{M})$, AZD8186 (250 nM), or Veh for 7 days. h Western blot showing levels of pAKT and pS6 in LNCaP-EV (empty vector) or LNCaP-p110 $\alpha$ WT (ectopic expression of DDK-tagged p110 ${ }^{\mathrm{WT}}$ ) cells cultured in regular 10\% FBS-RPMI treated with BYL719 $(1 \mu \mathrm{M})$, AZD8186 (250 nM), or Veh for $4 \mathrm{~h}$. Source data are provided as Source data file. All assays were performed with three biological replicates. ${ }^{\star \star \star \star} p<0.0001$, n.s: not significant, e: one-way ANOVA compared to BYL719 group, g: one-way ANOVA compared to AZD8186 group, error bar represents mean values \pm SD. 
a

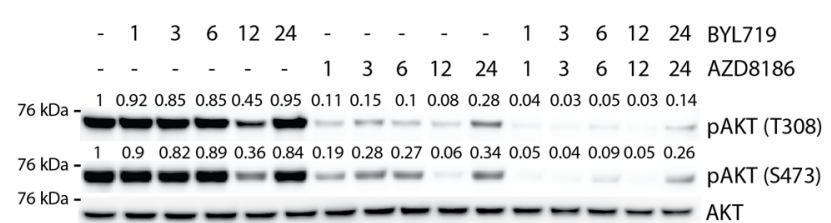

C

MSK-PCa3

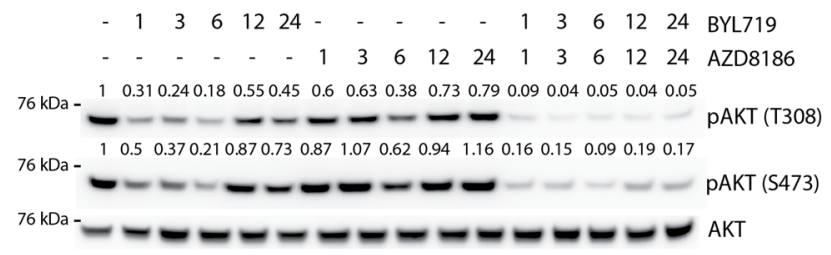

e

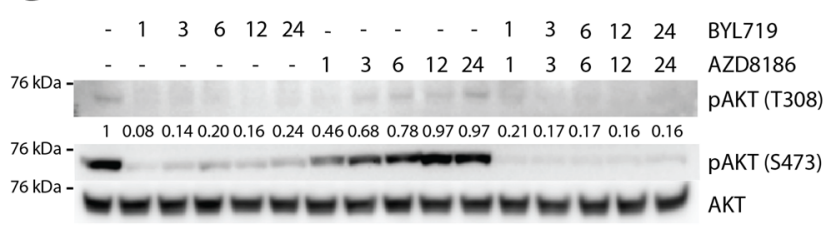

g

CWR22Pc Tumor

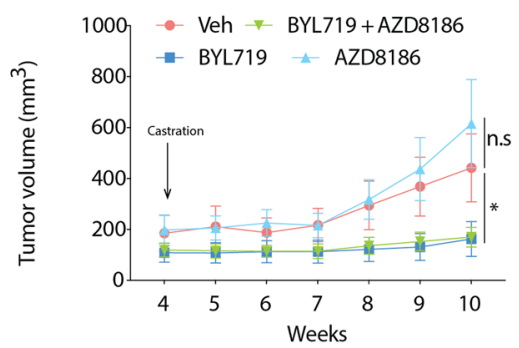

b
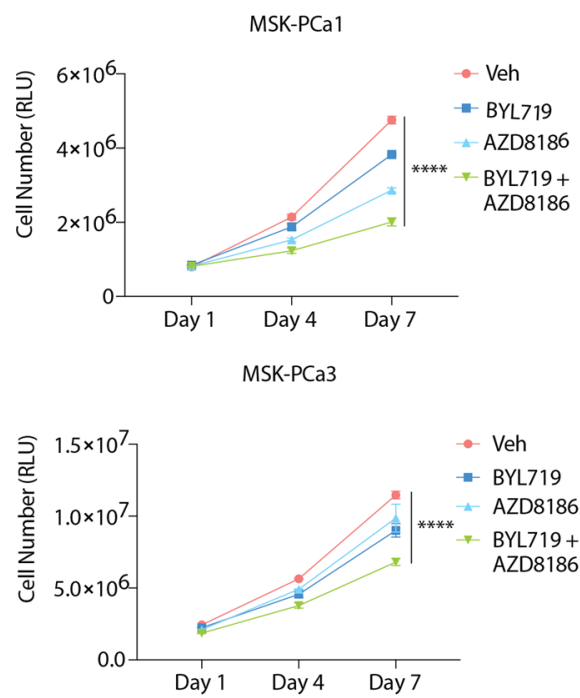

$\mathbf{f}$

CWR22PC-sgPTEN

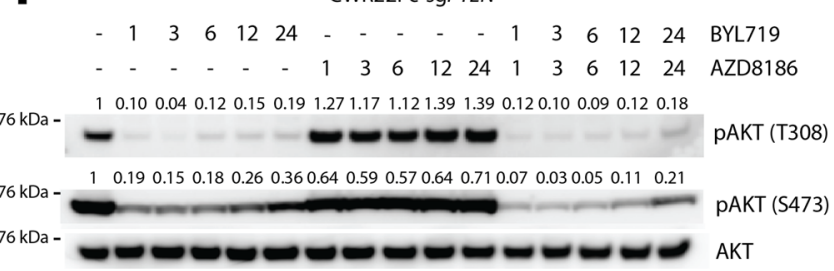

h CWR22PC Tumor

Vehicle BYL719 AZD8186 BYL719+

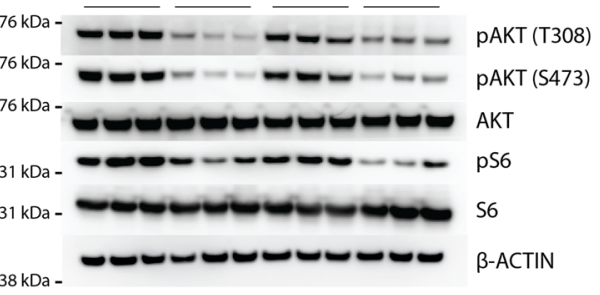

Fig. 3 PTEN-deficiency enhances relief of feedback inhibition through p110 $\alpha$ and p110 $\beta$ signaling following contralateral isoform selective inhibition. a, c Western blot showing pAKT levels in PDO models MSK-PCa1 and MSK-PCa3 treated with BYL719 (1 $\mu$ M), AZD8186 (250 nM), BYL719 + AZD8186, or Veh for $0,1,3,6,12$, and $24 \mathrm{~h}$, respectively. Quantification of pAKT473 and pAKT308 normalized to total AKT. b, d Growth assay of PDO lines MSK-PCa1 and MSK-PCa3 treated with BYL719 $(1 \mu \mathrm{M})$, AZD8186 (250 nM), BYL719 + AZD8186, or Veh. Cell number was read using CellTiter-Glo assay on Day 1, Day 4, and Day 7, respectively. e, f Western blot showing pAKT levels in CWR22Pc-sgNT or CWR22Pc-sgPTEN isogenic pairs treated with BYL719 (1 $\mu$ M), AZD8186 (250 nM), BYL719 + AZD8186, or Veh for 0, 1, 3, 6, 12, and $24 \mathrm{~h}$, respectively. Quantification of pAKT473 and pAKT308 normalized to total AKT. $g$ Growth of CWR22Pc tumors in SCID mice treated with BYL719 (25 mg/kg), AZD8186 (75 mg/kg), BYL719 + AZD8186, or Veh. Mice were castrated when tumor reaches $\sim 200 \mathrm{~mm}^{3}$. h Western blot showing pAKT and pS6 levels in CWR22Pc tumors from (g). Source data are provided as Source data file. All assays were performed with three biological replicates. ${ }^{\star \star \star \star} p<0.0001,{ }^{\star} p=0.0142$, n.s: not significant, b, d, g: one-way ANOVA compared to Veh group, error bar represents mean values \pm SD, $\mathbf{g}$ : error bar represents mean values \pm SEM.

Despite the dramatic difference in PI3K rebound signaling between the two models, combined inhibition of p110 $\alpha+$ p $110 \beta$ reduced proliferation in vitro compared to single isoform inhibition in both models (Supplementary Fig. 3G, H). However, in vivo studies of PTEN wild-type, PIK3CA-mutant CWR22Pc demonstrated that these models are exquisitely sensitive to castration $+\mathrm{p} 110 \alpha$ inhibition, with no added therapeutic benefit to combined p110 $\beta$ inhibition, likely due to the lack of robust relief of feedback inhibition when PTEN is intact (Fig. 3g, h). This data demonstrate that loss of PTEN promotes relief of feedback inhibition and rebound signaling through alternative p110 isoforms and that in the setting of wild-type PTEN relief of feedback inhibition is minimal. Taken together, these findings have important clinical implications, as approximately 5\% of metastatic CRPC patients harbor activating PIK3CA alterations in the setting of wild-type PTEN and thus may benefit from p110aspecific inhibition combined with AR targeting therapies, similar to that reported in breast cancer patients in the SOLAR1 Trial.

Given the frequent co-occurrence of $P I K 3 C A / P I K 3 C B$ alterations and loss of PTEN in prostate cancer, we evaluated how these concomitant alterations impact $\mathrm{PI} 3 \mathrm{~K}$ feedback reactivation. In LNCaP-p110a ${ }^{\mathrm{E} 545 \mathrm{~K}}$, feedback PI3K reactivation was seen following treatment with BYL719 (p110a), while dual inhibition of both isoforms significantly reduced the level of pAKT (Supplementary 
Fig. 3B). Meanwhile, neither single isoform-selective inhibitor at standard dosing suppressed the growth of these mutant cells, inhibition of both isoforms was able to reduce cell proliferation (Supplementary Fig. 3A). Given that $\mathrm{p} 110 \alpha^{\mathrm{E} 545 \mathrm{~K}}$ was exogenously overexpressed at high levels, we also tested the efficacy of an increased concentration of BYL719 and when combined with AZD8186 resulted in the most potent anti-proliferative effect (Supplementary Fig. 3A). Similarly, in LNCaP-p110 $\beta^{\mathrm{E} 1051 \mathrm{~K}}$, feedback PI3K reactivation was observed following AZD8186 (p110 $\beta)$ treatment, while dual inhibition of both isoforms significantly repressed pAKT level (Supplementary Fig. 3F). In the same model, AZD8186 as a single agent was effective in suppressing growth of mutant cells probably due to the cell line's inherent dependency on $110 \beta$, but inhibition of both isoforms most effectively suppressed cell growth (Supplementary Fig. 3E).

Upregulation of IGF1R mediates resistance to p110 $\alpha+p 110 \beta$ dual blockade. Across our collection of PTEN-deficient PDOs, we observed that some of them exhibit exquisite sensitivity to dual inhibition of both PI3K isoforms, while others displayed innate resistance (Fig. 4a, b). When we compared their genomic profiles (MSK-IMPACT), we did not find distinct differences in the common oncogenic drivers between the two groups. We then shifted our attention to differential PI3K signaling mediators including differential RTK expression which has been implicated in altering responsiveness to PI3K inhibitors ${ }^{34-36}$. Exploratory RTK transcriptomic analysis across our organoid models revealed that IGF1R was upregulated in our resistant PDO MSK-PCa12 as well as in a PTEN wild-type PDO MSK-PCa15 (Supplementary Fig. 4A, B, Supplementary Data 3). We validated this finding at the protein level across our PDO models and found a correlation between IGF1R protein level and its activity (pIGF1R) (Supplementary Fig. 4C). Importantly, we observed significantly higher IGF1R/INSR activity in the resistant line MSK-PCa12, as compared to the sensitive line MSK-PCa11 (Fig. 4c). Higher IGF1R/ INSR activity was also associated with residual levels of the AKT targets pPRAS40 and downstream pS6 when p110 $\alpha+$ p110 $\beta$ dual inhibition was applied (Fig. 4c). This link suggests that residual PI3K activity may contribute to resistance following p110a+ p1 $10 \beta$ dual inhibition. Based on this finding, we hypothesized that genetic ablation of IGF1R may eliminate residual level of PI3K activity and re-sensitize PDOs that are resistant to dual inhibition of $\mathrm{p} 110 \alpha$ and $\mathrm{p} 110 \beta$. We first tested this hypothesis by knocking-down IGF1R in resistant MSK-PCa12 organoids using three independent shRNA hairpins against IGF1R. In comparison to shRenilla control, shRNA knockdown of IGF1R reduced AKT activity in Veh-treated condition and further suppressed AKT signaling (shown by pPRAS40 and pS6 levels) when combined with $\mathrm{p} 110 \alpha+\mathrm{p} 110 \beta$ dual inhibition in MSK-PCa12 (Fig. 4d). More importantly, shIGF1R restored sensitivity to p110 $\alpha+$ p110 $\beta$ dual inhibition in MSK-PCa12 (Fig. 4e). These findings were validated in vivo where knockdown of IGF1R synergized with PI3K inhibition in MSK-PCa12 tumor growth and pathway inhibition (Fig. 4f, g). Meanwhile, knockdown of INSR had a mild effect. This result is consistent with prior knowledge that INSR is more relevant to metabolic control rather than cell proliferation ${ }^{37}$ (Supplementary Fig. 5A, B).

We then tested if pharmacological inhibition of IGF1R with $\mathrm{p} 110 \alpha+\mathrm{p} 110 \beta$ dual inhibition would achieve similar therapeutic efficacy compared to genetic knockdown. As expected, the IGF1R/INSR dual inhibitor linsitinib plus BYL719 (p110a inhibitor) + AZD8186 (p110 $\beta$ inhibitor) was able to further decrease PI3K signaling and repress cell proliferation in MSKPCa12 (Supplementary Fig. 5C, D). To validate these findings, we knocked out PTEN in another PDO model, MSK-PCa15, which has high levels of endogenous IGF1R. Following PTEN knockout, this line displayed resistance to combined p110 $\alpha+\mathrm{p} 110 \beta$ inhibition, and sensitivity could be restored with linsitinib (Supplementary Fig. 5E-G). We next sought to ask if pharmacologic IGF1R inhibition could restore sensitivity of PI3K inhibition in vivo in the resistant MSK-PCa12 xenograft. Initial in vivo experiments testing the combination of BYL719+ AZD8186 + linsitinib displayed significant toxicity necessitating study termination (Supplementary Fig. 5H, I), so the experimental design was changed to evaluate the combination of linsitinib with BKM120, a pan-PI3K inhibitor. Upon 14 days post treatment, we already observed a significant anti-tumor effect in the combination condition; however, significant toxicity remained, resulting in dramatic weight loss requiring study termination for mice receiving linsitinib plus BKM120 (Fig. 4h, i). Collectively, these results suggest upregulation of IGF1R mediates residual PI3K activity and promotes resistance to $\mathrm{p} 110 \alpha+\mathrm{p} 110 \beta$ dual inhibition in PTEN-deficient prostate tumors. While these pathways are both therapeutically actionable, the toxicity observed limits the use of the combination of currently available clinical grade inhibitors.

AKT-selective inhibition overcomes resistance caused by RTK upregulation. AKT is one of the dominant downstream signaling components of the PI3K signaling pathway ${ }^{7}$. However, directly targeting AKT using ATP-competitive inhibitors was challenging due to the high similarities of ATP-binding pockets among AGC kinase family members ${ }^{38}$. Recently, two newly developed AKTselective inhibitors have advanced in clinical trials of several tumor types ${ }^{39-43}$. We chose to study the Genentech compound ipatasertib as it is currently in a phase III clinical trial for mCRPC in combination with $\mathrm{AR}$ pathway inhibition based on our previous work.

To characterize the effects of ipatasertib in our model systems, we performed pharmacological dynamic studies both in vitro and in vivo using the LNCaP cell line to determine the optimum dose for target inhibition. We observed sufficient target inhibition (shown by the levels of pPRAS40 and pS6) at $500 \mathrm{nM}$ in vitro and at $50 \mathrm{mg} / \mathrm{kg}$ in vivo (Supplementary Fig. 6A, B). Based on the results in the LNCaP model, we hypothesized that directly targeting AKT may allow us to overcome the RTK-mediated resistance to $\mathrm{PI} 3 \mathrm{~K}$ inhibition observed in our models. Indeed, for the BYL719 + AZD8186 resistant line MSK-PCa12, ipatasertib efficiently reduced downstream PI3K-AKT activity and achieved a potent anti-proliferative effect in vitro, combined with enzalutamide (Fig. 5a, b). Also, when we tested an independent PDO model, MSK-PCa15, which displays high levels of IGF1R and becomes resistance to $\mathrm{p} 110 \alpha+\mathrm{p} 110 \beta$ dual inhibition upon PTEN deletion, the MSK-PCa15-sgPTEN cells similarly showed sensitivity to ipatasertib (Supplementary Fig. 6C, D). Moreover, ipatasertib combined with enzalutamide was more effective at suppressing tumor growth than dual p110a/p110 $\beta$ inhibition and enzalutamide in MSK-PCa12 xenograft models (Fig. 5c and Supplementary Fig. 6E).

Given the recent positive interim analysis of the phase III ipatasertib and abiraterone clinical trial in mCRPC patients with PTEN-loss, there has been discussion on whether AKT inhibition should be extended to patients harboring other activating alterations of the PI3K pathway, such as PIK3CA mutations. To address this question, CWR22Pc xenograft models were treated with ipatasertib or BYL719 with or without castration. Surprisingly, AKT inhibition combined with castration in this molecular context had minimal effect and was inferior to BYL719+ castration (Fig. 5d). Also, AKT inhibition significantly increased MAPK signaling compared with p110a inhibition (Fig. 5e). To 
a

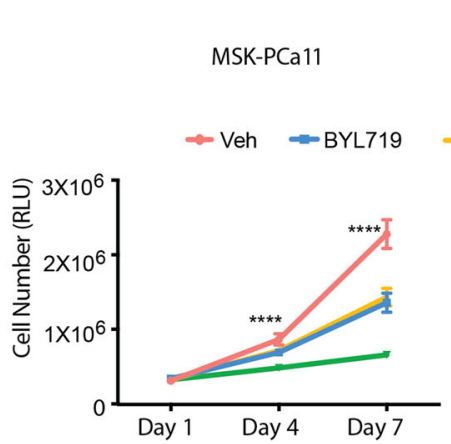

d

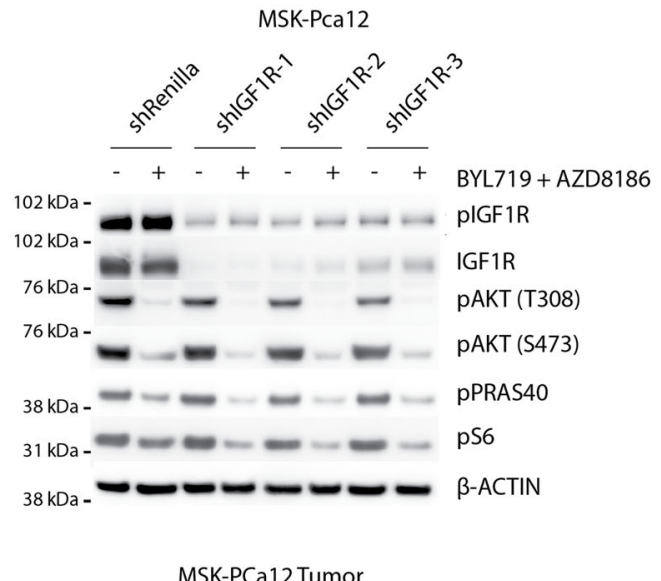

MSK-PCa12 Tumor

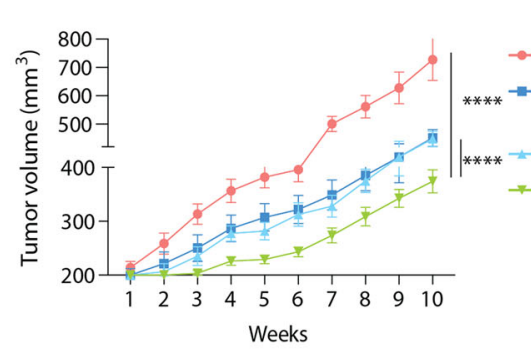

h

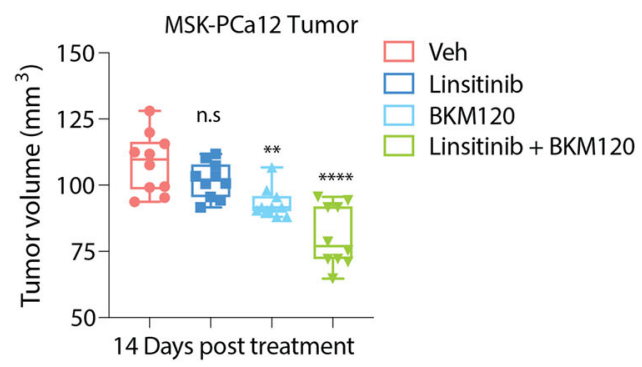

directly compare the efficacy of PI3K to AKT inhibition in hormone-regulated cancers, we evaluated a panel of prostate and breast cancer models harboring alterations in the PI3K signaling pathway. In the setting of wild-type PTEN where PI3K signaling was activated through PIK3CA mutation or RTK signaling, PI3K pathway inhibition with $\mathrm{p} 110 \alpha+\mathrm{p} 110 \beta$ was generally more antiproliferative than AKT inhibition (Fig. 5f). However, in the setting of PTEN-loss (endogenous or isogenic PTEN knockout pairs), AKT inhibition showed greater efficacy at suppressing cell growth than PI3K inhibition even when p110a activating mutations were present (Fig. 5g). This association was especially significant for the breast cancer cell lines harboring mutant
C

MSK-PCa12

- BYL719+AZD8186

$$
102 \mathrm{kDa}-+++ \text { BYL719 + AZD8186 }
$$

$\quad=-\mathrm{plGF1R/INSR}$

$76 \mathrm{kDa}-\ldots \quad \ldots \quad$ PAKT (T308)

$76 \mathrm{kDa}-\ldots \quad$ - $\quad$ PAKT (S473)

$76 \mathrm{kDa}-\ldots-$ AKT

$38 \mathrm{kDa}--m-$ pPRAS40

$38 \mathrm{kDa}----1$ PRAS40

$31 \mathrm{kDa}-$ - - pS6

$31 \mathrm{kDa}-2-\infty \mathrm{S} 6$

$38 \mathrm{kDa}---\infty$-ACTIN

\section{e}

MSK-PCa12

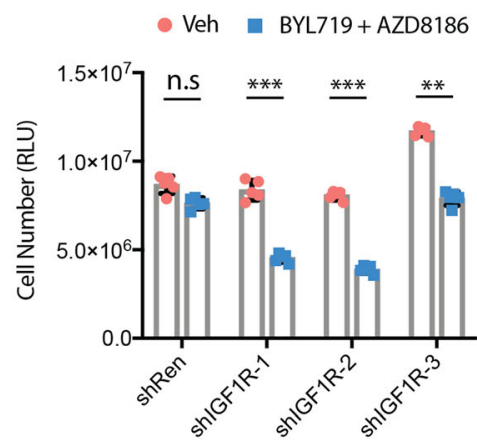

$\mathbf{g}$

MSK-PCa12 Tumor

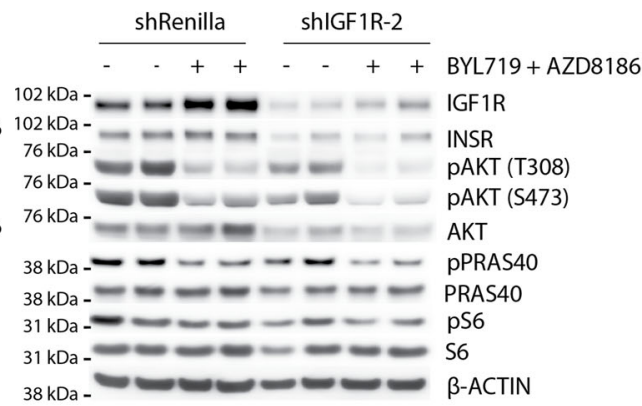

i

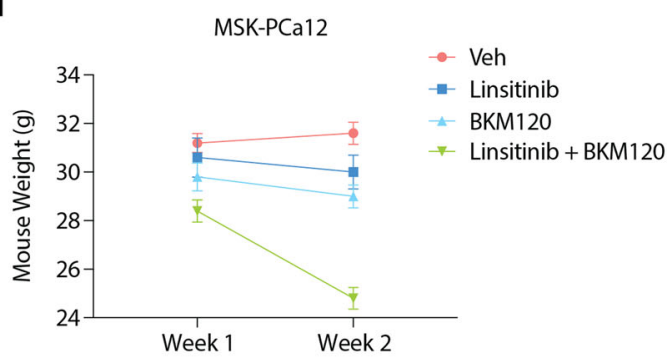

PIK3CA when PTEN was exogenously knocked out (Fig. 5g, EFM19, T47D, MCF-7, MDA-MB-453, and BT20). In PTEN-loss, PIK3CA wild-type cells, AKT inhibition performed similar to PI3K inhibition with the exception of the organoids that harbored hyperactive RTK signaling where AKT inhibition was superior (Fig. 5h, MSK-PCa12, MSK-PCa15-sgPTEN, and MSK-PCa16sgPTEN).

Collectively, our data demonstrate that in tumors harboring loss of PTEN, inhibiting AKT may be more efficacious than targeting PI3K, as AKT inhibitors are effective independent of RTK levels or PIK3CA mutations. Conversely, in PIK3CA-mutant PTEN wild-type prostate and breast cancers, p110a inhibition 
Fig. 4 Upregulation of IGF1R mediates resistance to $\mathbf{p} \mathbf{1 1 0} \boldsymbol{\alpha}+\mathbf{p} \mathbf{1 1 0} \boldsymbol{\beta}$ dual blockade. $\mathbf{a}$, $\mathbf{b}$ Growth assay of PDO lines MSK-PCa11 and MSK-PCa12 treated with BYL719 (1 $\mu$ M), AZD8186 (250 nM), BYL719 + AZD8186 or Veh. Cell number was read using CellTiter-Glo assay on Day 1, Day 4, and Day 7, respectively. c Western blot showing levels of IGF1R/INSR and AKT signaling in PDO lines MSK-PCa11 and MSK-PCa12 treated with BYL719

$(1 \mu \mathrm{M})+$ AZD8186 $(250 \mathrm{nM})$ or Veh for $4 \mathrm{~h}$. d Western blot showing levels of IGF1R and AKT signaling in MSK-PCa12-shRenilla or MSK-PCa12-shIGF1R lines treated with BYL719 $(1 \mu \mathrm{M})+$ AZD8186 $(250 \mathrm{nM})$ or Veh for $4 \mathrm{~h}$. e CellTiter-Glo assay showing cell viability of MSK-PCa12-shRenilla or MSK-PCa12shIGF1R lines treated with BYL719 $(1 \mu \mathrm{M})+$ AZD8186 $(250 \mathrm{nM})$ or Veh, Day 7. f Growth of MSK-PCa12-shRenilla or MSK-PCa12-sh/GF1R tumors in SCID mice treated with BYL719 $(25 \mathrm{mg} / \mathrm{Kg})+$ AZD8186 $(75 \mathrm{mg} / \mathrm{Kg})$ or Veh $(n=5$ mice per group). $\mathbf{g}$ Western blow showing levels of INSR, IGF1R, pAKT, pPRAS40, and pS6 in MSK-PCa12-shRenilla or MSK-PCa12-shIGF1R tumors from (f), 14 days post treatment. $\mathbf{h}$ Volume of MSK-PCa12 tumors in SCID mice treated with linsitinib ( $40 \mathrm{mg} / \mathrm{kg}$ ), BKM120 $(50 \mathrm{mg} / \mathrm{kg}$ ), linsitinib + BKM120, or Veh, 14 days post treatment, box and whiskers Min to Max.all points ( $n=10$ mice per group). i Body weight analysis of mice in $(\mathbf{h})(n=10$ mice per group). Source data are provided as Source data file. All assays were performed with three biological replicates. ${ }^{\star \star \star \star} p<0.0001,{ }^{\star \star \star} p=0.0003,{ }^{\star \star} p=0.0041$, n.s: not significant, $\mathbf{a}, \mathbf{b}$ : multiple $t$-test two-sided, e, $\mathbf{h}$ : one-way ANOVA compared to Veh group, f: one-way ANOVA compared to shIGF1R group treated with BYL719+AZD8186, a, b, e: error bar represents mean values $\pm S D, \mathbf{f}, \mathbf{h}$ : error bar represents mean values \pm SEM, i: error bar represents $\pm S D$.

provides superior antiproliferative effects. Furthermore, as we have previously published, due to reciprocal feedback inhibition between the PI3K and AR pathways, combined inhibition of these pathways is required in prostate cancer (Fig. 5i).

\section{Discussion}

Targeting PI3K alterations to treat human malignancies has been proposed for more than a decade, yet previous clinical trials using PI3K pathway inhibitors against multiple human malignancies did not generate profound success due to significant toxicities and low efficacy ${ }^{9,10}$. Recently, improved understanding of the differential expression and functional divergence amongst PI3K isoforms raised the hope that targeting individual p110 isoforms may maximize efficacy and minimize toxicity ${ }^{11,15}$. For example, a p110 $\delta$-selective inhibitor idelalisib was recently approved to treat several types of blood malignancies including chronic lymphocytic leukemia, relapsed follicular lymphoma, and relapsed small lymphocytic lymphoma ${ }^{44-46}$. In a more recent phase III clinical trial (SOLAR-1), BYL719, a p110 $\alpha$-selective inhibitor combined with fulvestrant significantly improved progression-free survival in ER-positive, PIK3CA-mutant breast cancers $^{26}$. Because PIK3CA (p110 ) and PIK3CB (p110ß) are ubiquitously expressed in tumors of epithelial origin ${ }^{8,11}$, understanding $\mathrm{p} 110 \alpha$ or $\mathrm{p} 110 \beta$ dependency across different genetic backgrounds is critical for selectively targeting the PI3K signaling axis in PI3K pathwayaltered tumors.

In metastatic prostate cancer, $\mathrm{PI} 3 \mathrm{~K}$ pathway alterations are seen in more than $50 \%$ of patients, among which more than $80 \%$ harbor PTEN loss-of-function alterations. Limited evidence suggesting that PI3K signaling in PTEN-deficient tumors is more dependent on $\mathrm{p} 110 \beta$ led to the development of several phase I clinical trials evaluating p110 $\beta$-selective inhibitors in PTENdeficient mCRPC patients ${ }^{14,28}$. Here we characterized an expanded cohort of patient-derived organoid (PDO) models that harbor loss of PTEN to define PI3K isoform dependency. Surprisingly, we found that PTEN-status is not the determinant driver of p110 isoform selectivity. While the mechanisms determining p110 isoform dominance in PTEN-deficient prostate cancer remain unknown, this finding has important clinical implications that loss of PTEN alone will not be a biomarker of p110 $\beta$ inhibition sensitivity.

This unexpected finding raises the question about what molecular mechanisms drive p110 isoform selectivity. Indeed, through our genomic analyses of $\mathrm{mCRPC}$, we found frequent occurrence of $P I K 3 C A / P I K 3 C B / P I K 3 R 1$ alterations in the setting of both PTEN wild-type and PTEN loss. Through the modeling of $P I K 3 C A$ or $P I K 3 C B$ alterations using multiple PDO and cell line models, we discovered that activating mutations of p110a or p110 $\beta$ can drive or shift isoform dependency. This finding indicates that 110 isoform-activating mutations could be a potential biomarker for the selection of an isoform-selective inhibitor, especially in the context of PIK3CA mutant, PTEN wild-type prostate cancer. Our results provide support for the development of clinical trials evaluating combined p110a (BYL719) and AR inhibition in patients with $\mathrm{mCRPC}$ harboring PIK3CA-mutant, PTEN wild-type cancers.

For the first time, we show that PI3K relief of feedback inhibition is robust and therapeutically meaningful in the context of PTEN-loss but not in the setting of wild-type PTEN. In prostate cancers harboring loss of PTEN, this feedback inhibition is reciprocal between the p110 isoforms and occurs in the context of p110a or p110 $\beta$-dominant signaling. Although dual inhibition of $\mathrm{p} 110 \alpha+\mathrm{p} 110 \beta$ can efficiently block PI3K relief of feedback inhibition and achieve potent anti-tumor effect in some prostate cancers harboring loss of PTEN, we also identified that resistance to $\mathrm{p} 110 \alpha+\mathrm{p} 110 \beta$ inhibition can occur through IGF1R-mediated PI3K residual activity. While addition of an IGF1R inhibitor, linsitinib, restored $\mathrm{p} 110 \alpha+\mathrm{p} 110 \beta$ sensitivity in resistant lines, this combination is associated with significant toxicity limiting clinical development. Importantly, we show that the use of an AKT-selective inhibitor such as ipatasertib (GDC-0068) is not only able to overcome this resistance, but generally performs superior to pan-PI3K inhibition with lower toxicity across PTENdeficient pre-clinical models. Moreover, these results are also supported by a recent phase II and phase III clinical trial for mCRPC patients in which treatment with ipatasertib plus abiraterone has achieved a significantly longer RPFS in PTEN-deficient patients ${ }^{25}$. Furthermore, in the metastatic breast cancer, PTEN alterations are shown to confer resistance to p110aselective inhibition, demonstrating the need for an alternative strategy to target PI3K signaling ${ }^{33}$. Our results suggest AKT inhibitors may also be superior to pan-PI3K inhibition in breast and prostate cancers harboring loss of PTEN, however in the context of PIK3CA mutant PTEN wild-type cancers there is limited efficacy to AKT inhibition and targeting p110a is superior, secondary to dual suppression of downstream PI3K and MAPK signaling. Importantly, PIK3CA mutant PTEN wild-type prostate cancers should not be broadly grouped with PTEN loss cancers in future AKT inhibitor clinical trials. Taken together, as we gain further insights into the mechanisms driving p110 isoform dependency and validate isoform selective biomarkers, therapeutic trials may be developed to selectively target a specific isoform through a precision medicine approach.

\footnotetext{
Methods

Patient-derived prostate cancer organoids and cell lines. Patient-derived organoids (PDOs) (MSK-PCa1, MSK-PCa2, MSK-PCa3, MSK-PCa8, MSK-PCa11, MSK-PCa12, MSK-PCa15, MSK-PCa16) were obtained from Dr. Yu Chen's laboratory through collaboration. MSK-PCa1, MSK-PCa2, MSK-PCa3, and MSK PCa8 were previously published by Dr. Yu Chen's group ${ }^{30}$. MSK-PCa11, MSKPCa12, MSK-PCa15, and MSK-PCa16 were newly generated and can be obtained
} 


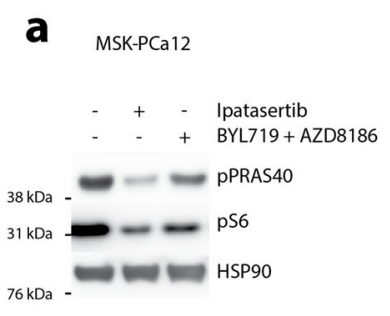

d

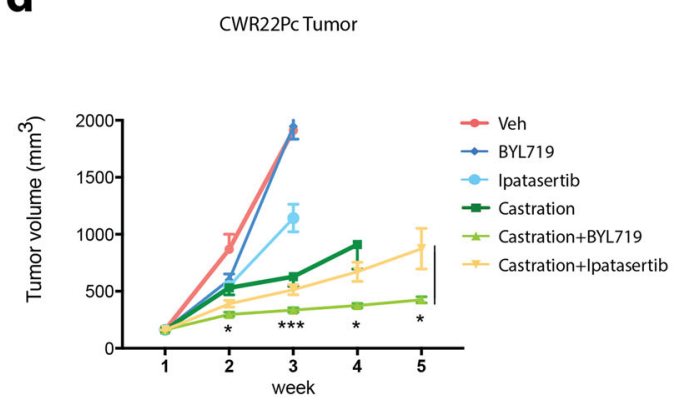

b

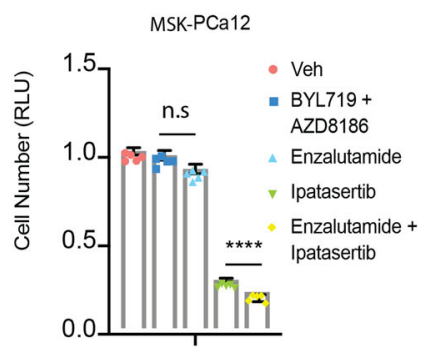

C

C MSK-PCa12 Tumor

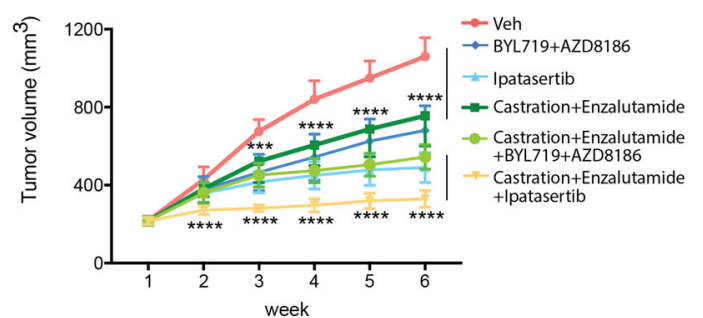

e

CWR22Pc Tumor

Castration Castration

Vehicle Castration +BYL719 +|patasertib

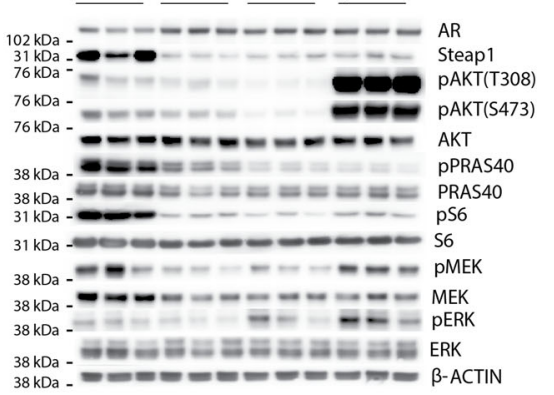

h

g

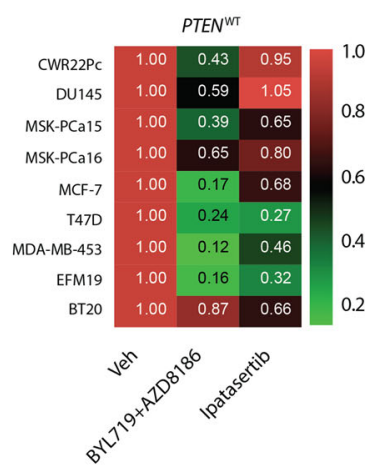

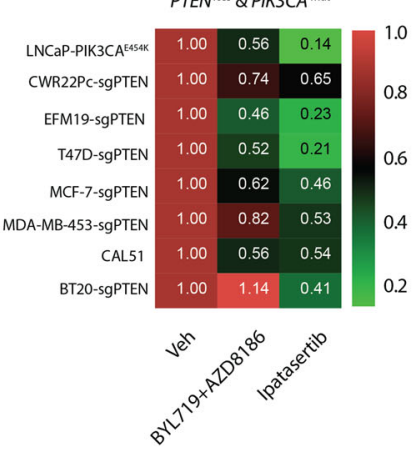

PTEN ${ }^{\text {loss }} \&$ PIK3CA WT

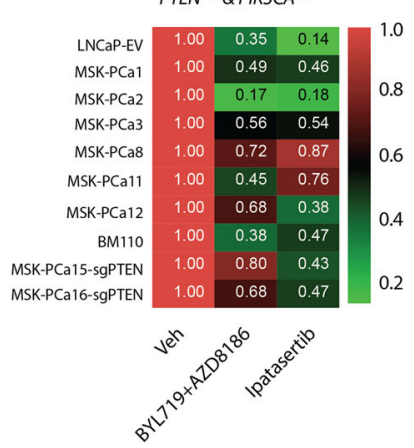

i

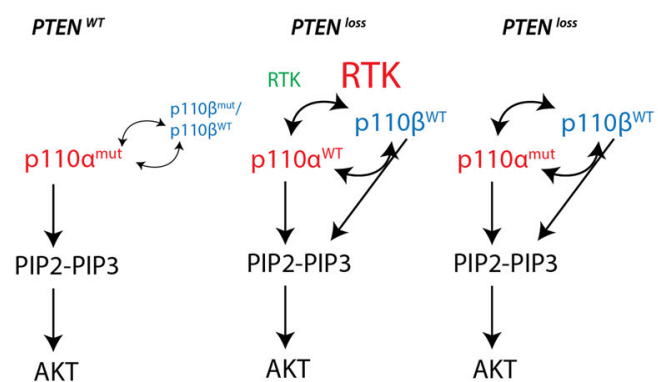

BYL719 (p110a)

Low RTK: BYL719 (p110a)

+ AZD 8186(p110ß)

or ipatasertib (AKT)
High RTK: ipatasertib (AKT)

ipatasertib (AKT)

from Dr. Yu Chen's group upon request. PDOs were maintained and cultured in standard organoid culture condition ${ }^{47}$. LNCaP (\#ATCC ${ }^{\circledR}$ CRL-1740 ${ }^{\mathrm{Tm}}$ ) and DU145 (\#ATCC ${ }^{\circledR}$ HTB-81 ${ }^{\mathrm{TN}}$ ) were obtained from American Type Culture Collection (ATCC, Manassas, VA). MCF7(\#ATCC ${ }^{\circledR}$ HTB-22 $^{\text {px }}$ ), T47D (\#ATCC ${ }^{\circledR}$ CRL-2865 $^{\text {rx }}$ ), MDA-MB-453(\#ATCC ${ }^{\circledR}$ HTB-131 $^{\mathrm{mw}}$ ), EFM19(DSMZ, \#ACC-231), CAL51(DSMZ, \#ACC-231) and BT20 (\#ATCC ${ }^{\circledR}$ HTB-19 ${ }^{\text {tut }}$ ) cell lines were gifts from Dr. Guotai Xu (MSKCC). CWR22Pc was a gift from Marja T. Nevalainen (Thomas Jefferson University, Philadelphia, PA), and CWR22Pc-EP was generated and maintained as previously described ${ }^{48,49}$. Cell lines were maintained in RPMI (LNCaP, CWR22Pc T47D, EFM19, CAL-51) or DMEM (MCF7, MDA-MB-453, and BT20). Additional supplements for all cell lines were 10\% FBS (Omega Scientific, Tarzana, CA),

PenStrep (1\%), L-glutamine (1\%), sodium pyruvate (1\%), and HEPES $\mathrm{pH}=7.6$

(1\%). All cell lines and prostate cancer organoids used in our studies have tested negative for mycoplasma using the MycoProbe Mycoplasma Detection Kit (R\&D Systems) within one month of initiating experiments. All cell lines and organoids were freshly thawed and only passaged to achieve the number of cells required for 
Fig. 5 AKT-selective inhibition overcomes resistance caused by RTK upregulation. a Western blot showing levels of AKT signaling in MSK-PCa12 organoids treated with ipatasertib $(500 \mathrm{nM})$ or BYL719 $(1 \mu \mathrm{M})+$ AZD8186 $(250 \mathrm{nM})$ for $4 \mathrm{~h}$. b CellTiter-Glo assay showing cell viability of MSK-PCa12 organoids treated with BYL719 $(1 \mu \mathrm{M})+$ AZD8186 $(250 \mathrm{nM})$, enzalutamide $(10 \mu \mathrm{M})$, ipatasertib $(500 \mathrm{nM})$, enzalutamide + ipatasertib, or Veh, Day 7 . c Growth of MSK-PCa12 tumors in SCID mice treated with enzalutamide (10 mg/kg), ipatasertib (50 mg/kg), BYL719 (25 mg/kg) + AZD8186 (75 mg/kg), ipatasertib + enzalutamide, BYL719 + AZD8186 + enzalutamide or Veh. Some of mice were castrated when tumor reached $\sim 200 \mathrm{~mm}{ }^{3}(n=5 \mathrm{mice}$ per group) ${ }^{* *} p$-value $=0.0011$. d Growth of CWR22Pc tumors in SCID mice treated with BYL719 $(25 \mathrm{mg} / \mathrm{kg})$, ipatasertib (50 mg/ $\left.\mathrm{kg}\right)$ or Veh. Some of mice were castrated when tumor reaches $200 \mathrm{~mm}^{3}$ ( $n=5$ mice per group) Castration + BYL719 vs. Castration + Ipatasertinib, week 2: $p$-value $=0.045$, week 3: $p$-value $=0.0086$, week 4: $p$-value $=0.012$, week 5: $p$-value $=0.046$. e Western blot showing PI3K/AKT/MAPK signaling in CWR22Pc tumors from (d). f-h Heat map showing drug sensitivity of ipatasertib $(500 \mathrm{nM})$ or BYL719 $(1 \mu \mathrm{M})+$ AZD8186 $(250 \mathrm{nM})$ on day 7, in cells characterized as PTEN wild type with PIK3CA mutants or RTK hyperactive (f), PIK3CA mutants with PTEN-loss (g), and PIK3CA wild type with PTEN-loss (h). Genetically manipulated lines are isogenic pairs. i Mechanism model of PI3K pathway alterations and response to clinically available therapeutic agents. All assays were performed with three biological replicates. ${ }^{\star \star \star \star} p<0.0001,{ }^{\star \star \star} p<0.001,{ }^{\star \star} p<0.01,{ }^{\star} p<0.05$, n.s: not significant, $\mathbf{b}$ : one-way ANOVA compared to Veh group, error bar represents mean values \pm SD. $\mathbf{c}$, $\mathbf{d}$ : multiple $t$-test, two-sided error bar represents mean values \pm SEM.

in vitro or in vivo experiments. Cell lines and organoids developed by our group were validated by STR genotyping protocols.

Cell proliferation assay. $5 \times 10^{3}$ cells in $100 \mu \mathrm{L}$ media were seeded per well in a 96-well plate. Drugs were administered the next day to a total volume of $200 \mu \mathrm{L}$ of media per well, and media and drugs were replaced every $48 \mathrm{~h}$ to maintain their efficacy. The number of viable cells was measured using CellTiter-Glo Luminescent Cell Viability Assay kit (Promega, G7573). Luminescence was detected using a Veritas Microplate Luminometer (Turner Biosystems). Luminescence signal representing relative number of cells was recorded as RLU (relative light units) according to manufacturer's instruction. CellTiter-Glo 2.0 reagent was aliquoted into working solutions and stored at $-80^{\circ} \mathrm{C}$, then at each assay time point, thawed to room temperature. Equal volume of reagent was added to wells of 96 -well plates using a multi-channel pipette. Plates were incubated in room temperature on an orbital shaker for $10 \mathrm{~m}$ to stabilize the reaction. All cell proliferation assays were repeated in at least two independent biological replicates.

Stable gene expression analysis. pLenti-Lentiviral vector (pLenti-C-Myc-DDKP2A-Neo) (Origene, PS100081) was used to express PIK3CA wild-type (Origene, RC213112), PIK3CA mutant (E545K) (Origene, RC400348), and PIK3CB wild-type (Origene, RC215433). PIK3CB mutant (E1051K) was obtained through sitedirected mutagenesis with plenti-PIK3CB vector. The PI3KCB cDNA mutant was generated following manufacture's instruction using QuikChange II XL SiteDirected Mutagenesis Kit, 10 Rxn (Agilent, 200521). Inducible PTEN expression lentivector was obtained as a gift from Dr. Wouter Karthaus (MSKCC). Doxycycline $(500 \mathrm{ng} / \mathrm{mL})$ was added to LNCaP-EV (empty vector) or LNCaP-PTEN cells for $8 \mathrm{~h}$ for PTEN induction. LNCaP-EV cells serve as control. All expression vectors were sequenced verified.

Therapeutic agents. The AR inhibitor MDV3100, enzalutamide, was synthesized by the MSKCC chemistry core. MDV3100 was used in our studies in vitro at a concentration of $10 \mathrm{mmol} / \mathrm{L}$ and in vivo with a dose of $10 \mathrm{mg} / \mathrm{kg} / \mathrm{day}^{29}$. The PI3K p110aselective inhibitor BYL719 was purchased from LC Laboratories (cat\# A-4477). BYL719 was dissolved in $0.5 \%$ methyl cellulose and dosed at $25 \mathrm{mg} / \mathrm{kg}$ daily P.O. The PI3K p110ß-selective inhibitor AZD8186 was kindly provided by Laboratory of Neal Rosen and purchased from SelleckChem (cat\# S7694). AZD8186 was dissolved in 0.5\% HPMC $+0.1 \%$ Tween 80 and dosed at $75 \mathrm{mg} / \mathrm{kg}$ b.i.d P.O. Pan-PI3K inhibitor BKM120 was purchased from SelleckChem (cat\# S2247). BKM120 was dissolved in 0.5\% methyl cellulose and dosed at $50 \mathrm{mg} / \mathrm{kg}$ daily P.O. IGF1R/INSR inhibitor linsitinib was purchased from LC Laboratory (cat\# L-5814). Linsitinib was dissolved in $25 \mathrm{mM}$ Tartaric Acid and dosed at $40 \mathrm{mg} / \mathrm{kg}$ daily P.O. AKT inhibitor ipatasertib was provided by Genentech under an MTA and was dissolved in 0.5\% Methylcellulose and 0.2\% Tween80 for in vivo study.

Mouse xenograft procedure. $2 \times 10^{6}$ cells resuspended in $100 \mu \mathrm{L}$ of $1: 1 \mathrm{mix}$ of growth media and matrigel (Corning, 356237) were engrafted or injected into 6 to 8 -week-old CB17-SCID male mice (Taconic). Tumor volume was measured weekly with Peira TM900 (Peira Scientific Instruments) and was calculated using the formula: Volume 1/4 Length $\times$ Width $\times$ Height. A total of 5 mice (10 tumors) per group were used to assay tumor growth in vivo in response to treatment. Treatment was initiated when tumor volume reached $\sim 200 \mathrm{~mm}^{3}$. All experiments were approved by our IACUC protocol 06-07-012.

Immunoblot. Cell lysates were prepared in RIPA buffer supplemented with proteinase and phosphatase inhibitors. Proteins were resolved on NuPAGE Novex 4-12\% Bis-Tris Protein Gels (Thermo Fisher Scientific) and transferred electronically onto a PVDF $0.45 \mathrm{~mm}$ membrane (Millipore). Membranes were blocked in $5 \%$ BSA diluted in Tris buffer saline plus $0.1 \%$ Tween 20 (TBST) for $1 \mathrm{~h}$ at room temperature and were incubated with primary antibodies in $5 \%$ BSA at $4{ }^{\circ} \mathrm{C}$ overnight. After 3 washes of $10 \mathrm{~m}$ in TBST, membranes were incubated with secondary antibodies in 5\% BSA at room temperature. All immunoblots were performed with three independent biological repeats. Representative and consistent findings were shown.

Antibodies. The following antibodies were used for Western blotting: AR (Abcam, ab108341), $\beta$-actin (Cell Signaling Technology, 4970S, 1:10,000), phospho-AKT (Ser473) (Cell Signaling Technology, 4060L), phospho-AKT (Thr308) (Cell Signaling Technology, 4056S), AKT (Cell Signaling Technology, 4685S), phospho-S6 ribosomal protein (Cell Signaling Technology, 4856S), S6 (5G10) ribosomal protein (Cell Signaling Technology, 2217S), PTEN (Cell Signaling Technology, 9188L), phospho-PRAS40 (Thr246) (Cell Signaling Technology, 2997S), PRAS40 (Cell Signaling Technology, 2691S), phospho-IGF1R (Tyr1135/1136)/INSR (Tyr1150/ 1151) (Cell Signaling Technology, 3024S), phospho-IGF1R (Tyr1135) (Cell Signaling Technology, 3918S), IGF1R (Cell Signaling Technology, 9750S), INSR (Cell Signaling Technology, 3025S), phospho-p44/42 MAPK (Erk1/2) (Thr202/Tyr204) (D13.14.4E) XP (Cell Signaling Technology, 4370S), p44/42 MAPK (Erk1/2) (137F5) (Cell Signaling Technology, 4695S), phospho-MEK1/2 (Ser217/221) (41G9) (Cell Signaling Technology, 9154S), MEK1/2 (D1A5) (Cell Signaling Technology, 8727S), PI3K kinase P110a (Cell Signaling Technology, 4249S), PI3K kinase P110ß (Cell Signaling Technology, 3011S), HSP90 (Cell Signaling Technology, 4875S, 1:10,000), Cyclophilin B (Cell Signaling Technology, 43603S, 1:10,000), DDK tag (Origene, TA50011-100). All western blot antibodies were used at a 1:1000 dilution unless specificied.

CRISPR/Cas9-mediated knock-out. To knock out PTEN in PDOs, human prostate and breast cancer cell lines, sgRNA against human PTEN exon 1 was designed (https://zlab.bio/guide-design-resources/) and cloned into the LentiCRISPRv2 backbone (Addgene, 52962). Lentiviruses for sgRNAs were generated in $293 \mathrm{~T}$ cells by standard methods using Lipofectamine 2000 (Invitrogen, 11668500). Cell lines were infected with lentivirus for 48 to $72 \mathrm{~h}$ and selected with puromycin $(1 \mu \mathrm{g} / \mathrm{mL})$ for 7 days. The target guides sequences are listed in Supplementary data 2 .

MirE-based gene knockdown. The lentiviral (SGEP, LEPC) miR-E-based shRNA expression vectors were generously gifted by Dr. Johannes Zuber (Research Institute of Molecular Pathology, Vienna, Austria ${ }^{50}$. The target guides sequences are listed in Supplementary data 2

RNA-Sequencing and analysis. RNA was isolated from organoids with low passage number (less than 5) using Qiagen RNeasy kits. Library preparation and RNA sequencing were performed by MSKCC Genomics Core Laboratory using Illumina HiSeq with 50 or 75 bp paired-end reads and $\sim 30$ million reads were generated for each sample. The reads were mapped human genome reference sequence (GRC37/hg19) using STAR (v2.3) ${ }^{51}$ and quantified into Transcripts Per Kilobase Million (TPM) using Cufflinks with upper quartile normalization (v2.1 ${ }^{52}$. Heatmaps analysis were generated using R-studio (package pheatmap 1.0.12). RTK normalized expression values are available in Supplementary data 1.

Study approval. All experiments involving animals were approved by MSKCC Animal Ethics Committee (Judith Farber).

Reporting summary. Further information on research design is available in the Nature Research Reporting Summary linked to this article.

\section{Data availability}

The RNAseq data performed on the patient derived prostate cancer organoids was deposited to GEO (accession number GSE181374), and can be accessed at: https:// 
www.ncbi.nlm.nih.gov/geo/query/acc.cgi?acc=GSE181374. The processed RNAseq data are also available for analysis through cBioPortal For Cancer Genomics (https:// www.cbioportal.org) using the link: https://www.cbioportal.org/study/summary? id=prad_mskcc_cheny1_organoids_2014.

Received: 23 December 2020; Accepted: 5 August 2021; Published online: 20 August 2021

\section{References}

1. Sanchez-Vega, F. et al. Oncogenic signaling pathways in the cancer genome atlas. Cell 173, 321-337.e310 (2018).

2. Samuels, Y. et al. High frequency of mutations of the PIK3CA gene in human cancers. Science 304, 554 (2004).

3. Abida, W. et al. Genomic correlates of clinical outcome in advanced prostate cancer. Proc. Natl Acad. Sci. USA 116, 11428-11436 (2019).

4. Robinson, D. et al. Integrative clinical genomics of advanced prostate cancer. Cell 162, 454 (2015).

5. Ciriello, G. et al. Comprehensive molecular portraits of invasive lobular breast cancer. Cell 163, 506-519 (2015).

6. Cancer GenomeAtlas, N. Comprehensive molecular portraits of human breast tumours. Nature 490, 61-70 (2012).

7. Fruman, D. A. et al. The PI3K pathway in human disease. Cell 170, 605-635 (2017).

8. Engelman, J. A. Targeting PI3K signalling in cancer: opportunities, challenges and limitations. Nat. Rev. Cancer 9, 550-562 (2009).

9. Heudel, P. E. et al. Phase II study of the PI3K inhibitor BKM120 in patients with advanced or recurrent endometrial carcinoma: a stratified type I-type II study from the GINECO group. Br. J. Cancer 116, 303-309 (2017).

10. Armstrong, A. J. et al. Phase II trial of the PI3 kinase inhibitor buparlisib (BKM-120) with or without enzalutamide in men with metastatic castration resistant prostate cancer. Eur. J. Cancer 81, 228-236 (2017).

11. Liu, P., Cheng, H., Roberts, T. M. \& Zhao, J. J. Targeting the phosphoinositide 3-kinase pathway in cancer. Nat. Rev. Drug Disco. 8, 627-644 (2009).

12. Yuzugullu, H. et al. A PI3K p110beta-Rac signalling loop mediates Pten-lossinduced perturbation of haematopoiesis and leukaemogenesis. Nat. Commun. 6, 8501 (2015).

13. Schmit, F. et al. PI3K isoform dependence of PTEN-deficient tumors can be altered by the genetic context. Proc. Natl Acad. Sci. USA 111, 6395-6400 (2014).

14. Ni, J. et al. Functional characterization of an isoform-selective inhibitor of PI3K-p110beta as a potential anticancer agent. Cancer Disco. 2, 425-433 (2012).

15. Jia, S., Roberts, T. M. \& Zhao, J. J. Should individual PI3 kinase isoforms be targeted in cancer? Curr. Opin. Cell Biol. 21, 199-208 (2009)

16. Wee, S. et al. PTEN-deficient cancers depend on PIK3CB. Proc. Natl Acad. Sci. USA 105, 13057-13062 (2008)

17. Zhao, J. J. et al. The p110alpha isoform of PI3K is essential for proper growth factor signaling and oncogenic transformation. Proc. Natl Acad. Sci. USA 103, 16296-16300 (2006).

18. Yang, Q., Modi, P., Newcomb, T., Queva, C. \& Gandhi, V. Idelalisib: first-inclass PI3K delta inhibitor for the treatment of chronic lymphocytic leukemia, small lymphocytic leukemia, and follicular lymphoma. Clin. Cancer Res. 21, 1537-1542 (2015).

19. Hancox, U. et al. Inhibition of PI3Kbeta signaling with AZD8186 inhibits growth of PTEN-deficient breast and prostate tumors alone and in combination with docetaxel. Mol. Cancer Ther. 14, 48-58 (2015).

20. Fritsch, C. et al. Characterization of the novel and specific PI3Kalpha inhibitor NVP-BYL719 and development of the patient stratification strategy for clinical trials. Mol. Cancer Ther. 13, 1117-1129 (2014).

21. Toska, E. et al. PI3K pathway regulates ER-dependent transcription in breast cancer through the epigenetic regulator KMT2D. Science 355, 1324-1330 (2017).

22. Rathkopf, D. E. et al. Everolimus combined with gefitinib in patients with metastatic castration-resistant prostate cancer: Phase $1 / 2$ results and signaling pathway implications. Cancer 121, 3853-3861 (2015).

23. Bosch, A. et al. PI3K inhibition results in enhanced estrogen receptor function and dependence in hormone receptor-positive breast cancer. Sci. Transl. Med. 7, 283 ra251 (2015).

24. Carver, B. S. et al. Reciprocal feedback regulation of PI3K and androgen receptor signaling in PTEN-deficient prostate cancer. Cancer Cell 19, 575-586 (2011).

25. de Bono, J. S. et al. Randomized phase II study evaluating Akt blockade with ipatasertib, in combination with abiraterone, in patients with metastatic prostate cancer with and without PTEN loss. Clin. Cancer Res. 25, 928-936 (2019).
26. Andre, F. et al. Alpelisib for PIK3CA-mutated, hormone receptor-positive advanced breast cancer. N. Engl. J. Med. 380, 1929-1940 (2019).

27. Juric, D. et al. Phosphatidylinositol 3-kinase alpha-selective inhibition with alpelisib (BYL719) in PIK3CA-altered solid tumors: results from the first-inhuman study. J. Clin. Oncol. 36, 1291-1299 (2018).

28. Jia, S. et al. Essential roles of PI(3)K-p110beta in cell growth, metabolism and tumorigenesis. Nature 454, 776-779 (2008)

29. Zhang, Z. et al. Loss of CHD1 promotes heterogeneous mechanisms of resistance to AR-targeted therapy via chromatin dysregulation. Cancer Cell 37, 584-598 e511 (2020).

30. Gao, D. et al. Organoid cultures derived from patients with advanced prostate cancer. Cell 159, 176-187 (2014).

31. Schwartz, S. et al. Feedback suppression of PI3Kalpha signaling in PTENmutated tumors is relieved by selective inhibition of PI3Kbeta. Cancer Cell 27, 109-122 (2015).

32. Costa, C. et al. Measurement of PIP3 levels reveals an unexpected role for p110beta in early adaptive responses to p110alpha-specific inhibitors in luminal breast cancer. Cancer Cell 27, 97-108 (2015).

33. Juric, D. et al. Convergent loss of PTEN leads to clinical resistance to a $\mathrm{PI}(3)$ Kalpha inhibitor. Nature 518, 240-244 (2015).

34. Zorea, J. et al. IGF1R upregulation confers resistance to isoform-specific inhibitors of PI3K in PIK3CA-driven ovarian cancer. Cell Death Dis. 9, 944 (2018).

35. Hopkins, B. D. et al. Suppression of insulin feedback enhances the efficacy of PI3K inhibitors. Nature 560, 499-503 (2018).

36. Leroy, C. et al. Activation of IGF1R/p110beta/AKT/mTOR confers resistance to alpha-specific PI3K inhibition. Breast Cancer Res. 18, 41 (2016)

37. Sasaoka, T. et al. Comparison of the insulin and insulin-like growth factor mitogenic intracellular signaling pathways. Endocrinology 137, 4427-4434 (1996).

38. Lin, J. et al. Targeting activated Akt with GDC-0068, a novel selective Akt inhibitor that is efficacious in multiple tumor models. Clin. Cancer Res 19 1760-1772 (2013)

39. Schmid, P. et al. Capivasertib plus paclitaxel versus placebo plus paclitaxel as first-line therapy for metastatic triple-negative Breast cancer: the PAKT trial. $J$. Clin. Oncol. 38, 423-433 (2020).

40. Banerji, U. et al. A phase I open-label study to identify a dosing regimen of the Pan-AKT inhibitor AZD5363 for evaluation in solid tumors and in PIK3CAmutated breast and gynecologic cancers. Clin. Cancer Res. 24, 2050-2059 (2018).

41. Kim, S. B. et al. Ipatasertib plus paclitaxel versus placebo plus paclitaxel as first-line therapy for metastatic triple-negative breast cancer (LOTUS): a multicentre, randomised, double-blind, placebo-controlled, phase 2 trial. Lancet Oncol. 18, 1360-1372 (2017).

42. Yan, Y. et al. Evaluation and clinical analyses of downstream targets of the Akt inhibitor GDC-0068. Clin. Cancer Res. 19, 6976-6986 (2013).

43. Davies, B. R. et al. Preclinical pharmacology of AZD5363, an inhibitor of AKT: pharmacodynamics, antitumor activity, and correlation of monotherapy activity with genetic background. Mol. Cancer Ther. 11, 873-887 (2012).

44. Sharman, J. P. et al. Final results of a randomized, phase III study of rituximab with or without idelalisib followed by open-label idelalisib in patients with relapsed chronic lymphocytic leukemia. J. Clin. Oncol. 37, 1391-1402 (2019)

45. Jones, J. A. et al. Efficacy and safety of idelalisib in combination with ofatumumab for previously treated chronic lymphocytic leukaemia: an openlabel, randomised phase 3 trial. Lancet Haematol. 4, e114-e126 (2017).

46. Furman, R. R. et al. Idelalisib and rituximab in relapsed chronic lymphocytic leukemia. N. Engl. J. Med. 370, 997-1007 (2014).

47. Karthaus, W. R. et al. Identification of multipotent luminal progenitor cells in human prostate organoid cultures. Cell 159, 163-175 (2014)

48. $\mathrm{Mu}, \mathrm{P}$. et al. SOX2 promotes lineage plasticity and antiandrogen resistance in TP53- and RB1-deficient prostate cancer. Science 355, 84-88 (2017).

49. Zhang, Z. et al. Tumor microenvironment-derived NRG1 promotes antiandrogen resistance in prostate cancer. Cancer Cell 38, 279-296 e279 (2020).

50. Fellmann, C. et al. An optimized microRNA backbone for effective singlecopy RNAi. Cell Rep. 5, 1704-1713 (2013).

51. Dobin, A. et al. STAR: ultrafast universal RNA-seq aligner. Bioinformatics 29 , 15-21 (2013).

52. Roberts, A., Trapnell, C., Donaghey, J., Rinn, J. L. \& Pachter, L. Improving RNA-Seq expression estimates by correcting for fragment bias. Genome Biol. 12, R22 (2011).

\section{Acknowledgements}

A special thanks to members of the Chen, Sawyers, and Rosen labs for providing informative discussion. This work was funded in part through NIH/NCI Prostate SPORE P50-CA092629 (B.S. Carver, N. Rosen, C.L. Sawyers, Y. Chen), NIH/NCI U54-CA224079 (B.S. Carver, Y. Chen, C.L. Sawyers), NIH/NCI R01-CA182503 (B.S. Carver, N. Mao, and Y.S. Lee), PCF Challenge Award (B.S. Carver, Y. Chen, and A.C. Hsieh), NIH/NCI R37 CA230617 (A.C. Hsieh), and the MSKCC NIH/NCI Cancer Center Support Grant P30 
CA008748. Funding through the STARR Cancer Consortium (Y. Chen, B.S. Carver) allowed for establishment of a prostate organoid core to assist in our experiments. All authors approved the final manuscript. Z.Z. is supported by the NCI Predoctoral to Postdoctoral Fellow F99/K00 Transition Award (F99CA223063).

\section{Author contributions}

N.M., Z.Z., Y.C., A.C.S., and B.S.C. conceived the project. N.M., Z.Z., N.R., C.L.S., and B.S.C. oversaw the project, performed experimental design and data interpretation. Z.Z and B.S.C. co-wrote the manuscript. N.M., Y.L., and A.C.S. edited the manuscript. Z.Z., N.M., Y.L., D.C., A.A.R., X.C., Q.C., and E.D.S. performed in vivo experiments. Y.C., H.D., C.L., S.H., D.L., and G.X. assisted with the prostate cancer organoid model experiments. N.M., Z.Z., Y.L., and H.A.C. cloned plasmid reagents. N.M., Z.Z., and Y.L. performed western blots, in vitro growth assay, and qRT-PCR analysis. Z.Z., N.M., and B.S.C. performed analysis on cbioportal.org. All authors approved of the final manuscript.

\section{Competing interests}

C.L.S. is co-inventors of enzalutamide and apalutamide and may be entitled to royalties. C.L.S. serves on the Board of Directors of Novartis and is a co-founder of ORIC Pharm. $\mathrm{He}$ is a science advisor to Agios, Beigene, Blueprint, Column Group, Foghorn, Housey Pharma, Nextech, KSQ, Petra, and PMV. He was a co-founder of Seragon, purchased by Genentech/Roche in 2014. N.R. is on the scientific advisory board (SAB) and receives research funding from Chugai, on the $\mathrm{SAB}$ and owns equity in Beigene, and Fortress. N.R. is also on the SAB of Daiichi-Sankyo, Astra-Zeneca-MedImmune, and F-Prime, and is a past SAB member of Millenium-Takeda, Kadmon, Kura, and Araxes. N.R. is a consultant to Novartis, Boehringer Ingelheim, Tarveda, and Foresight and consulted in the last three years with Eli Lilly, Merrimack, Kura Oncology, Araxes, and Kadman. N.R. owns equity in ZaiLab, Kura Oncology, Araxes, and Kadman. N.R. collaborates with Plexxikon. A.C.H. has received research funding from eFFECTOR Therapeutics. The remaining authors declare no competing interests.

\section{Additional information}

Supplementary information The online version contains supplementary material available at https://doi.org/10.1038/s41467-021-25341-9.

Correspondence and requests for materials should be addressed to B.S.C.

Peer review information Nature Communications thanks Lucio Miele and the other anonymous reviewer(s) for their contribution to the peer review of this work. Peer reviewer reports are available.

Reprints and permission information is available at http://www.nature.com/reprints

Publisher's note Springer Nature remains neutral with regard to jurisdictional claims in published maps and institutional affiliations.

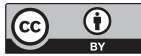

Open Access This article is licensed under a Creative Commons Attribution 4.0 International License, which permits use, sharing, adaptation, distribution and reproduction in any medium or format, as long as you give appropriate credit to the original author(s) and the source, provide a link to the Creative Commons license, and indicate if changes were made. The images or other third party material in this article are included in the article's Creative Commons license, unless indicated otherwise in a credit line to the material. If material is not included in the article's Creative Commons license and your intended use is not permitted by statutory regulation or exceeds the permitted use, you will need to obtain permission directly from the copyright holder. To view a copy of this license, visit http://creativecommons.org/ licenses/by/4.0/.

(C) The Author(s) 2021 